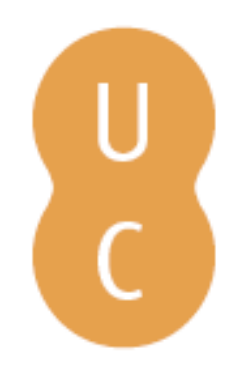

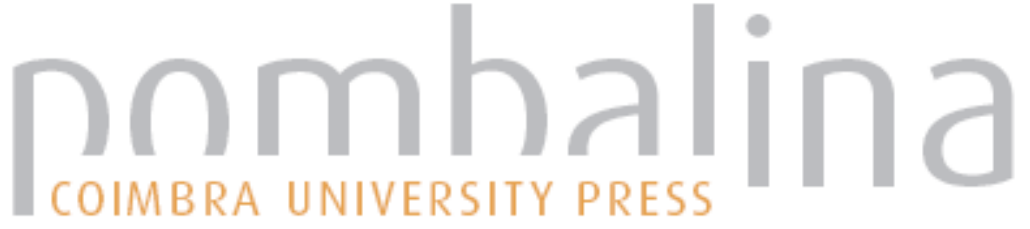

\section{Avaliação de desempenho das unidades de saúde para a melhoria dos cuidados de saúde primários}

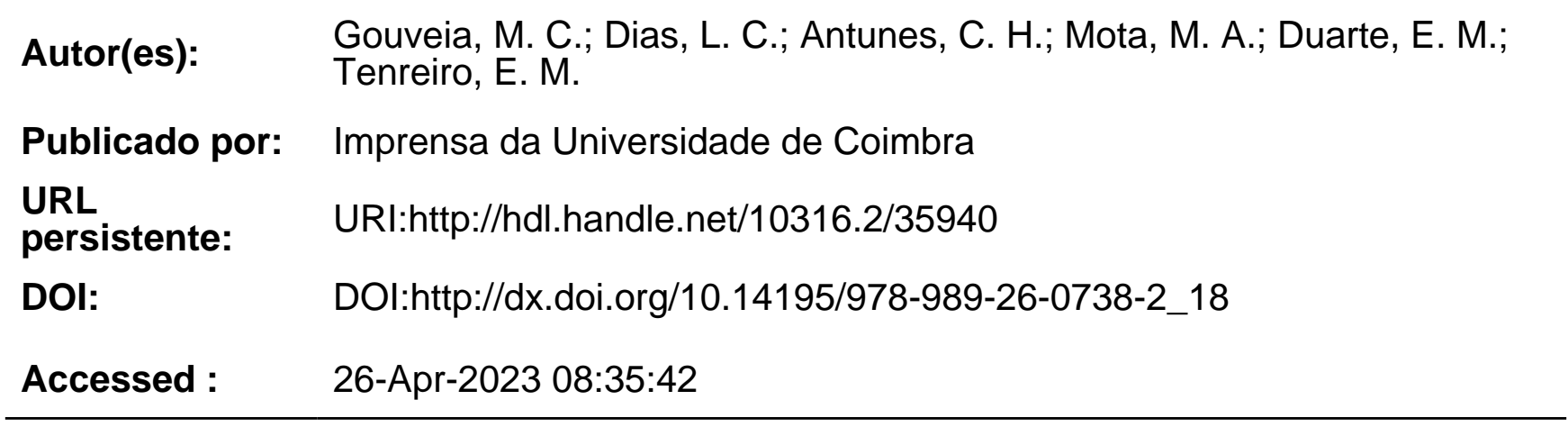

A navegação consulta e descarregamento dos títulos inseridos nas Bibliotecas Digitais UC Digitalis, UC Pombalina e UC Impactum, pressupõem a aceitação plena e sem reservas dos Termos e Condições de Uso destas Bibliotecas Digitais, disponíveis em https://digitalis.uc.pt/pt-pt/termos.

Conforme exposto nos referidos Termos e Condições de Uso, o descarregamento de títulos de acesso restrito requer uma licença válida de autorização devendo o utilizador aceder ao(s) documento(s) a partir de um endereço de IP da instituição detentora da supramencionada licença.

Ao utilizador é apenas permitido o descarregamento para uso pessoal, pelo que o emprego do(s) título(s) descarregado(s) para outro fim, designadamente comercial, carece de autorização do respetivo autor ou editor da obra.

Na medida em que todas as obras da UC Digitalis se encontram protegidas pelo Código do Direito de Autor e Direitos Conexos e demais legislação aplicável, toda a cópia, parcial ou total, deste documento, nos casos em que é legalmente admitida, deverá conter ou fazer-se acompanhar por este aviso.

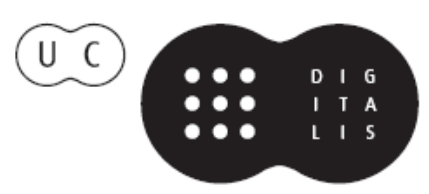




\section{INVESTIGAÇÃO OPERACIONAL EM AÇÃO \\ CASOS DE APLICAÇÃO}

RUI CARVALHO OLIVEIRA JOSÉ SOEIRO FERREIRA (EDITORES)

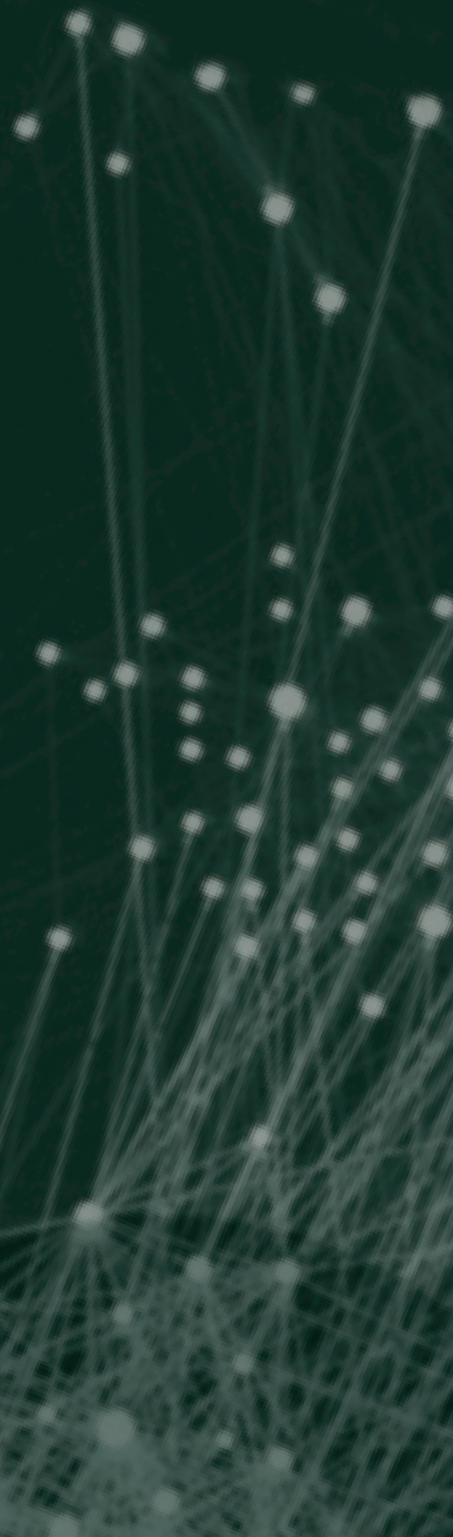




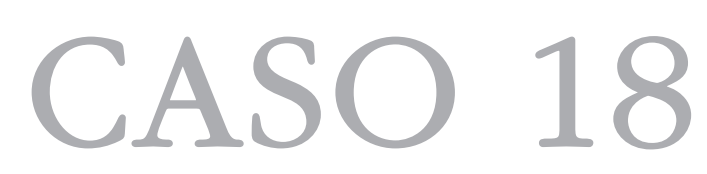

Avaliação de desempenho das UNIDADES DE SAÚDE PARA A MELHORIA DOS CUIDADOS DE SAÚde PRIMÁrios

M.C. Gouveia

IPC - ISCA Coimbra

INESC Coimbra

mgouveia@iscac.pt

L.C. Dias

Faculdade de Economia, Universidade de Coimbra INESC Coimbra

lmcdias@fe.uc.pt

C.H. Antunes

DEEC-FCT Universidade de Coimbra

INESC Coimbra

cantunes@inescc.pt

M.A. Mota

ACES Baixo Mondego I mariaaugustamota@gmail.com

E.M. Duarte ACES Baixo Mondego I emrduarte@gmail.com

E.M. Tenreiro ACES Baixo Mondego I Eduardo.tenreiro@gmail.com 


\section{RESUMO}

Este artigo relata um estudo de avaliação de desempenho de unidades de saúde prestadoras de cuidados de saúde primários realizado com o envolvimento dos decisores. Foram utilizadas metodologias, algumas desenvolvidas para este caso em particular, que exploram as ligações entre a Data Envelopment Analysis (DEA) e Análise de Decisão Multicritério (MultiCriteria Decision Analysis - MCDA), para incorporar preferências de gestão na avaliação de desempenho de doze unidades com dados relativos ao ano de 2010. Neste trabalho são apresentados dois modelos distintos, resultantes de discussões com um grupo de decisores, que combinam aspectos de avaliação como o acesso, os recursos utilizados, os serviços prestados e os resultados alcançados nos Cuidados de Saúde Primários. É utilizada uma variante do modelo DEA aditivo em duas fases para a avaliação de eficiência. Esta análise contribui para a identificação das melhores práticas, das fontes de ineficiência, bem como das diferenças em relação às melhores práticas. O interesse da abordagem reside não só na contribuição para conceber medidas corretivas adequadas baseadas nesses resultados, mas também na vertente de proporcionar informação relevante para a tomada de decisões mais fundamentadas sobre as metas dos indicadores de desempenho no futuro.

\section{PALAVRAS-CHAVE}

Data Envelopment Analysis; Análise de eficiência; Análise Multicritério; Cuidados de saúde primários. 


\section{Introdução}

Os Cuidados de Saúde Primários (CSP) são considerados de crucial importância no sistema de saúde (SNS) português. A experiência internacional demonstra que os modelos de organização e gestão dos CSP, bem como as estratégias de financiamento adotadas e a forma de articulação com os restantes níveis de cuidados, podem potenciar ou, pelo contrário, comprometer o cumprimento dos princípios basilares dos sistemas de saúde, bem como das metas definidas pela política de saúde ao nível da equidade, acesso, eficiência e qualidade dos cuidados.

A reforma dos CSP, iniciada em 2005, tem como propósito melhorar o acesso aos cuidados de saúde e a qualidade dos serviços prestados. Ao mesmo tempo, visa ganhos de eficiência, porquanto a dimensão do SNS é de tal ordem que práticas inadequadas podem causar enormes desperdícios. O impacto desta reforma está agora a ser testemunhado, o que justifica a necessidade de avaliar os seus resultados de modo a identificar as melhores práticas nos CSP.

O objetivo do presente trabalho é avaliar a eficiência das unidades de CSP monitorizadas pelo Agrupamento de Centros de Saúde (ACES) Baixo Mondego I. Esta avaliação pretende conciliar algumas das prioridades estabelecidas pelo Ministério da Saúde em 2011, em particular a secção sobre o "custo-efetividade, custo-benefício e custo-utilidade" que constam do mapa geral das necessidades de novos conhecimentos elaborado pela Coordenação Estratégica da Reforma dos CSP. Este trabalho envolve decisores reais participantes na avaliação de desempenho das unidades de CSP que monitorizam, recorrendo a uma nova abordagem. São apresentados neste texto apenas dois dos modelos analisados, que visam estabelecer uma ligação entre aspectos de avaliação como o acesso, os recursos utilizados, os serviços prestados e os resultados obtidos. A escolha dos fatores incluídos nos modelos foi resultado de discussões com o grupo de decisores (GD), constituído por profissionais do ACES Baixo Mondego I, com base no Plano de Desempenho (Coordenação Estratégica dos CSP [CECSP], 2010). A análise realizada utilizou dados relativos ao ano de 2010, compreendendo todas as 12 unidades prestadoras de cuidados de saúde que o ACES Baixo Mondego I monitoriza e acompanha. 
A contribuição deste trabalho para o ACES Baixo Mondego I reside, essencialmente, na capacidade de usar múltiplos fatores num modelo integrado, dando a possibilidade de identificar as melhores práticas, as fontes de ineficiência, bem como as diferenças para as melhores práticas. Esta abordagem proporciona informações no sentido de serem introduzidas medidas corretivas e ajuda a estabelecer as metas de desempenho para o futuro.

O estudo da avaliação de eficiência na prestação de CSP tem sido alvo da atenção de vários autores, especialmente no Reino Unido. Na maioria dos trabalhos publicados para avaliar a prestação de serviços de saúde e qualidade de atendimento constata-se que são utilizados indicadores de performance (IPs) (Donabedian, 1980; Baker, 1992; Campbell et al., 1998; McColl et al., 1998; Van den Hombergh et al., 1998; Proctor e Campbell, 1999). Contudo, os modelos de avaliação de desempenho que são baseados num conjunto de IPs para comparar as entidades conduzem a análises pobres, uma vez que os diferentes IPs são analisados individualmente. Thanassoulis et al. (1996) e Giuffrida et al. (2000) apontaram as desvantagens do uso de IPs na avaliação de desempenho e propuseram técnicas alternativas, tais como a Data Envelopment Analysis (DEA). A DEA é uma abordagem não paramétrica, baseada em programação linear (PL), para avaliar a eficiência relativa de unidades organizacionais (Decision Making Unit - DMUs) semelhantes, gerando um indicador global de eficiência para cada unidade em avaliação, com base nos inputs que consome e nos outputs que produz. Desde meados de 1980 que esta metodologia é muitas vezes escolhida para avaliar a eficiência dos serviços de saúde (para revisões mais abrangentes ver Hollingworth, 2003; Amado \& Dyson, 2008). Existem alguns estudos realizados sobre a eficiência na prestação de CSP que recorrem à técnica DEA (e.g., Huang \& McLaughlin, 1989; Linna et al., 2003; Kirigia et al., 2004; Rosenman \& Friesner, 2004; Rouse et al., 2011; Thanassoulis et al., 2012) e relativamente poucos estudos (SalinasJimenez \& Smith, 1996; Wagner et al. 2003, Amado \& Santos, 2009; Milliken et al., 2011; Nuti et al., 2011) incluem medidas de qualidade da assistência/atendimento na medição da eficiência. 
Apesar de, nos últimos anos, se terem realizado muitos estudos de avaliação de eficiência em diferentes áreas utilizando DEA, poucos incorporam na análise as preferências dos gestores/decisores (ver Thanassoulis et al., 2004). A estrutura matemática dos modelos clássicos DEA permite total liberdade na escolha dos coeficientes de ponderação (pesos) dos inputs e dos outputs. Logo, a solução pode gerar coeficientes de ponderação nulos para variáveis importantes e eventualmente relações entre as variáveis, resultantes do próprio contexto do estudo, podem não ser consideradas. Como consequência, os resultados atingidos podem ser criticados pelo decisor, ficando comprometida a sua confiança na metodologia. Para ultrapassar este problema utilizam-se restrições aos coeficientes de ponderação com o objetivo de incorporar a opinião do decisor (Allen et al., 1997). As restrições mais comuns são as baseadas em restrições de razões entre pesos, na qual são definidos os limites inferiores e superiores atribuídos às razões entre os coeficientes de ponderação de duas quaisquer variáveis. Estas restrições definem uma região preferida da fronteira, onde são observadas as melhores práticas do modelo de produção em avaliação.

Este trabalho utiliza o método de duas fases desenvolvido por Gouveia et al. (2008), que é uma variante do modelo DEA aditivo com projeções orientadas (Ali et al., 1995), recorrendo a conceitos desenvolvidos no âmbito da MCDA com informação imprecisa (Athanassopoulos \& Podinovski, 1997; Dias \& Clímaco, 2000). Com as DMUs a assumir o papel de alternativas a serem comparadas, neste método os fatores de input e output são convertidos em funções de utilidade de acordo com informação de preferências fornecida pelos decisores e as funções de utilidade aditiva são utilizadas para agregar as utilidades (marginais) associadas a cada alternativa, com base na Teoria da Utilidade Multi-Atributo (Multi-Attribute Utility Theory - MAUT) (ver Keeney \& Raiffa, 1976). Ao invés de deixar cada DMU escolher livremente os coeficientes de ponderação associados a estas funções, foram incluídas no modelo as preferências dos decisores no sentido de construir restrições aos coeficientes de ponderação seguindo alguns dos protocolos de diálogo recomendados (ver von Winterfeldt \& Edwards, 1986). Neste método de duas fases, a medida de eficiência atribuída a cada DMU tem um significado intuitivo, correspondendo 
a uma medida "min-max regret" (perda de utilidade). O modelo de Gouveia et al. (2008) foi neste estudo adaptado para incluir o conceito de super-eficiência (Gouveia et al., 2013), a fim de assinalar, entre as DMUs eficientes, quais o são com maior margem.

O método das duas fases de Gouveia et al. (2008), com as modificações para incluir o conceito de super-eficiência, é apresentado na secção 2. A secção 3 descreve brevemente o sistema de cuidados de saúde português. Na secção 4 são apresentados os dados, os modelos discutidos com o GD e os protocolos de diálogo utilizados para obter as suas preferências. Alguns resultados dos modelos propostos são exibidos e analisados na secção 5. A secção 6 destaca as propostas de melhoria para as unidades ineficientes e os novos desenvolvimentos do método apresentado na secção 2. As considerações finais são apresentadas na secção 7.

\section{O método das duas fases baseado no modelo DEA aditivo com incorporação} de preferências

As origens da DEA remontam aos trabalhos de Farrell (1957) sobre métodos para avaliação da produtividade, em que era reconhecida a necessidade de dispor de indicadores de eficiência global, e não de mera produtividade de fatores, que resultassem da combinação de múltiplos inputs e outputs. O primeiro modelo de DEA, assumindo rendimentos constantes à escala (Constant Returns to Scale - CRS), resultou do trabalho de Charnes, Cooper e Rhodes (Charnes et al., 1978), motivado pela avaliação de programas educacionais para estudantes desfavorecidos em escolas públicas dos Estados Unidos da América. A característica fundamental deste modelo (geralmente designado na literatura científica por modelo CCR ou Engineering Ratio) é, para cada entidade sob avaliação, a transformação da situação de múltiplos outputs / múltiplos inputs na situação de output virtual / input virtual. Para cada DMU, a razão entre o output virtual e o input virtual fornece uma medida de eficiência, a qual é função de um conjunto de multiplicadores que constituem as variáveis de um modelo de programação linear fraccionária (depois reduzido a um modelo de PL). As DMUs devem possuir um grau de controlo sobre os inputs consumidos e os outputs produzidos, ou seja, 
autonomia de gestão, operando num ambiente relativamente homogéneo. Neste modelo espera-se uma variação proporcional dos produtos a partir da alteração de recursos em todos os níveis de escala.

A extensão do modelo CCR para o caso de rendimentos variáveis à escala (Variable Returns to Scale - VRS), foi proposta em Banker, Charnes e Cooper (Banker et al., 1984), modelo designado por BCC, onde basicamente se introduz uma restrição que garante que cada DMU em estudo será comparada com uma combinação convexa das restantes DMUs. De acordo com Ozcan (1993), pode dizer-se que o modelo VRS é a escolha mais adequada quando se pretende comparar unidades de saúde de tamanhos muito diferentes entre si, de modo que as unidades tenham como referência as outras com perfil semelhante.

Os diferentes modelos DEA procuram determinar quais das $n$ DMUs formam a fronteira eficiente no sentido da eficiência de Pareto-Koopmans. Uma DMU será eficiente se, comparativamente às demais, tiver maior produção para quantidades fixas de recursos (orientação a outputs) e/ou utilizar menos recursos para gerar uma quantidade fixa de produtos (orientação a inputs). A DEA constrói uma fronteira empírica eficiente, ou "superfície envolvente" de máxima eficiência (Ali \& Seiford, 1993). Isto permite a identificação de unidades de referência (benchmarks) cujos valores de eficiência servem como modelo para as restantes unidades envolvidas pela fronteira de eficiência.

Charnes et al. (1985) propuseram o modelo DEA aditivo como uma alternativa para o modelo BCC, que também considera que os rendimentos podem ser variáveis à escala, mas não requer uma escolha entre modelo orientado a inputs e modelo orientado a outputs.

A distinção entre modelo DEA aditivo e os modelos orientados reside no facto destes últimos terem um mecanismo de projeção em duas etapas. Nos modelos orientados, numa primeira etapa todos os fatores são reduzidos ou aumentados na mesma proporção até à superfície envolvente, e numa segunda etapa são calculadas as folgas que dão o ponto projetado na fronteira eficiente. Por sua vez, o modelo DEA aditivo utiliza somente a segunda etapa. O valor obtido na primeira etapa de modelos orientados fornece uma medida da 
ineficiência, entre 0 e 1, para as DMUs ineficientes de acordo com a orientação utilizada.

Seja $\left\{D M U_{j}:(j=1, \ldots, n)\right\}$ o conjunto das $n$ DMUs a ser avaliadas. Cada DMU $j$ consome a quantidade $x_{i j}(i=1, \ldots, m)$ do input $i$ e produz a quantidade $y_{r j}(r=1, \ldots, p)$ do output $r$. O modelo aditivo a resolver para cada DMU tem a formulação primal (1):

$$
\begin{aligned}
& \min _{\lambda, s^{-}, s^{+}} z_{k}=-\left(\sum_{i=1}^{m} s_{i}^{-}+\sum_{r=1}^{p} s_{r}^{+}\right) \\
& \text {s.a: } \quad-\sum_{j=1}^{n} \lambda_{j} x_{i j}-s_{i}^{-}=-x_{i k}, \quad i=1, \ldots, m \\
& \quad \sum_{j=1}^{n} \lambda_{j} y_{r j}-s_{r}^{+}=y_{r k}, \quad r=1, \ldots, p \\
& \quad \sum_{j=1}^{n} \lambda_{j}=1 \\
& \quad \lambda_{j}, s_{i}^{-}, s_{r}^{+} \geq 0, \quad j=1, \ldots, n ; i=1, \ldots, m ; r=1, \ldots, p
\end{aligned}
$$

O modelo DEA aditivo não devolve uma medida para as DMUs ineficientes. Este modelo apenas mede o excesso de inputs $\left(s^{-}\right)$e o défice de outputs $\left(s^{+}\right)$, em que uma DMU $k$ está a operar quando comparada com as DMUs que operam na fronteira eficiente. Se o valor obtido for negativo, então a DMU em análise está a operar de forma ineficiente em alguns fatores. Este valor é simétrico da soma das distâncias em cada dimensão à superfície envolvente (distância $\mathrm{L}_{1}$ ).

O método de duas fases desenvolvido por Gouveia et al. (2008) é uma variante do modelo DEA aditivo com projeções orientadas (Ali et al., 1995), que incorpora as preferências do decisor utilizando conceitos da MAUT com informação imprecisa (Dias \& Clímaco, 2000).

O objetivo da MAUT é encontrar uma única expressão que represente a atratividade global de cada alternativa, de acordo com as preferências do decisor, tendo em consideração os múltiplos critérios. É também propósito desta teoria simplificar a construção de funções de utilidade multicritério deixando que o decisor foque a sua atenção num critério de cada vez, e posteriormente na agregação dos vários critérios, em vez de avaliar diretamente a utilidade global. 
A eliciação de preferências é realizada interativamente, em pequenos passos, de forma construtiva. von Winterfeldt e Edwards (1986) apresentam detalhadamente várias técnicas de questionar o decisor no sentido de construir as funções de utilidade marginais (parciais) consistentes com as respostas obtidas.

Seja $u_{c}\left(a_{j}\right)$ a utilidade de uma alternativa $a_{j}$ de acordo com o critério $c$ (c $=1, \ldots, q$ ). A função de utilidade aditiva agrega estas funções através de uma soma ponderada na qual são usados $q$ coeficientes de escala, $w_{1}, \ldots, w_{q}$ : $U\left(a_{j}\right)=\sum_{c=1}^{q} w_{c} u_{c}\left(a_{j}\right)$ (ver Keeney \& Raiffa, 1976). A quantidade $u_{c}\left(a_{j}\right)$ pode ser interpretada como uma medida de desempenho da alternativa $a_{j}$ no critério $c$. Estas funções são definidas para cada critério convertendo a escala de desempenhos originais na escala de utilidades [0,1]. Por sua vez, $w_{c}$ é o coeficiente de ponderação que reflete a importância da função $u_{c}(c=1, \ldots, q)$. Os coeficientes de ponderação são normalizados de forma a que $\sum_{c=1}^{q} w_{c}=1$, com $w_{c} \geq 0, \forall c=1, \ldots, q$, podendo ainda ser acrescentadas pelo decisor outras restrições, caso se pretenda impedir combinações de valores para estes coeficientes que não estejam alinhados com os objetivos da organização.

Este procedimento ultrapassa o problema das escalas associado ao modelo DEA aditivo, uma vez que todas as medidas de input e output são convertidas em utilidade. Além disso, os coeficientes de ponderação usados na agregação adquirem um significado específico: são os coeficientes de escala das funções de utilidade. Os coeficientes de ponderação são valores escolhidos por cada DMU, a fim de se tornar a melhor DMU (se possível) ou então no sentido de minimizar a diferença de utilidade para a melhor DMU, ao invés de serem fixados previamente como no modelo de Ali et al. (1995).

A teoria de decisão clássica oferece algumas regras (tais como max-min e min-max regret) que podem ser aplicadas a situações onde as funções de utilidade são incertas.

No desenvolvimento do método das duas fases utilizou-se a regra min-max regret, referente ao conceito de perda de utilidade de escolher uma alternativa face a outra. A ideia é determinar para cada alternativa o potencial "arrependimento" associado à sua escolha, observando quão melhor poderia outra alternativa ser. Para um dado vetor de coeficientes de ponderação, a 
perda de utilidade de escolher a alternativa $a_{j}$ em vez de outra alternativa $a_{j} \neq a_{k}$ é dada por (2):

$$
R\left(a_{k}, a_{j}\right)=\max _{j \neq k} U\left(a_{j}\right)-U\left(a_{k}\right)
$$

A perda de utilidade máxima associada a uma dada alternativa $a_{k}, R_{\max }\left(a_{k}\right)$, é dada pela máxima perda de oportunidade associada à escolha dessa alternativa (3):

$$
R_{\max }\left(a_{k}\right)=\max _{w}\left\{\left(\max _{j \neq k} U\left(a_{j}\right)\right)-U\left(a_{k}\right)\right\}
$$

A decisão ótima min-max regret minimiza a perda no pior caso de acordo com possíveis realizações do vetor de coeficientes $w$, i.e., alternativas com $R_{\max }\left(a_{k}\right)$ mínimo são preferidas. Assim, a medida de eficiência atribuída a cada DMU ganha um significado intuitivo: corresponde a uma medida "minmax regret" (perda de utilidade).

Considerando que as alternativas a avaliar de acordo com $q(q=m+p)$ critérios são as DMUs, definem-se $q$ funções de utilidade de modo a que o pior nível da escala tenha o valor 0 e o melhor nível da escala tenha o valor 1 . Por outras palavras, quanto melhor (neste caso menor) for o desempenho associado a um fator de input maior será a sua utilidade (mais próxima estará de 1); para o caso dos outputs, quanto maior for o desempenho maior será a sua utilidade. Assim, depois de todos os fatores terem sido convertidos em utilidades, passam a ser considerados como outputs a maximizar. Para cada alternativa (DMU), atendendo ao modelo aditivo MAUT, a utilidade obtida é dada por: $U\left(D M U_{j}\right)=\sum_{c=1}^{q} w_{c} u_{c}\left(D M U_{j}\right), \quad$ onde $\quad w_{c} \geq 0, \forall c=1, \ldots, q \quad$ e $\sum_{c=1}^{q} w_{c}=1$. Os coeficientes de escala $w_{1}, \ldots, w_{q}$ são os coeficientes de ponderação das funções de utilidade e traduzem as taxas de compensação (trade-offs) da utilidade do decisor entre os vários fatores.

Para atender ao conceito de super-eficiência, introduzido em DEA por Andersen e Petersen (1993), o método de duas fases de Gouveia et al. (2008) foi modificado no sentido de dar a possibilidade de discriminar as unidades eficientes (Gouveia et al., 2013). Assim, o problema (4) é resolvido (Fase 1): 
$\min _{d_{k}, w} d_{k}$

s.a: $\sum_{c=1}^{q} w_{c} u_{c}\left(D M U_{j}\right)-\sum_{c=1}^{q} w_{c} u_{c}\left(D M U_{k}\right) \leq d_{k}, j=1, \ldots, n ; j \neq k$

$\sum_{c=1}^{q} w_{c}=1$

$w_{c} \geq 0, c=1, \ldots, q$

O valor ótimo $d_{k}^{*}$ indica a distância definida pela diferença de utilidade para a melhor de todas as DMUs (excluindo a que está em avaliação).

O propósito desta abordagem é, para a DMU $k$, calcular o vetor $w$ de ponderação que minimiza a distância (a diferença de utilidade) da unidade em causa para a melhor (note-se que a melhor alternativa dependerá também de $w$ ), excluindo-se ela própria do conjunto de referência. Depois, no caso de ser uma DMU ineficiente ou fracamente eficiente, encontra um alvo (par) eficiente (Fase 2). Os detalhes deste processo são os seguintes:

Fase 1: Converter os fatores de input e output em escalas de utilidade. Calcular a medida de eficiência, $d_{k}^{*}$, de cada DMU, $k=1, \ldots, n$, e o vetor de ponderação correspondente.

Fase 2: Se $d_{k}^{*} \geq 0$, então resolver o problema "aditivo ponderado" (5), usando o vetor de ponderação ótimo resultante da Fase $1, w_{k}^{*}$, e determinar o correspondente ponto projetado da DMU em análise.

$$
\begin{array}{ll}
\min _{\lambda, s} z_{k}=-\sum_{c=1}^{q} w_{c}^{*} s_{c} \\
\text { s. } a: \quad \sum_{j=1, j \neq k}^{n} \lambda_{j} u_{c}\left(D M U_{j}\right)-s_{c}=u_{c}\left(D M U_{k}\right), \quad c=1, \ldots, q \\
\quad \sum_{j=1, j \neq k}^{n} \lambda_{j}=1 \\
\lambda_{j} \geq 0, s_{c} \geq 0, \quad j=1, \ldots, k-1, k+1, \ldots, n ; c=1, \ldots, q
\end{array}
$$

Se o valor ótimo $d_{k}^{*}$ da função objetivo em (4) é não positivo, então a DMU $k$ em avaliação é eficiente (se o valor ótimo for nulo a DMU poderá ser fracamente eficiente). Caso contrário, é ineficiente e $d_{k}^{*}$ é a diferença mínima de utilidade para a melhor DMU (ou seja, a DMU com maior utilidade global). Contudo, com a modificação feita no método original, é possível discriminar as unidades eficientes. As variáveis $\lambda_{1}, \ldots, \lambda_{j}, j=1, \ldots, k-1, k+1, \ldots, n$, definem uma combinação convexa das $n$ DMUs. As DMUs eficientes (podendo ser apenas uma) que definem a combinação convexa com $\lambda_{j}>0$ são chamadas de "pares" da DMU $k$ em avaliação. A combinação convexa corresponde a um 
ponto na fronteira eficiente, melhor do que a DMU $k$ com uma diferença de valor de $s_{c}$ (folga) em cada critério $c$.

O modelo (5) admite alvos ótimos alternativos, cada um dos quais correspondendo a uma forma diferente de anular o valor da diferença $d_{k}^{*}$. Esses alvos correspondem a diferentes projeções sobre a fronteira eficiente. Existe a possibilidade de escolher quais os fatores que se permite alterar e limitar a melhoria máxima que se pode exigir em cada fator para atingir o valor global de utilidade do alvo, através da inserção de restrições às folgas $s_{c}$. O objetivo é evitar a fixação de alvos irrealistas, permitindo que as propostas de melhoria das unidades ineficientes sejam concordantes com os requisitos do decisor.

\section{O sistema de saúde português - Cuidados de saúde primários}

Os CSP são a primeira e principal forma de contacto da população com o sistema de saúde. Em 1979, o SNS foi criado com o objectivo de defender o direito à saúde e o dever de defesa e proteção da saúde da população. O SNS forneceria um serviço nacional, universal, integral e gratuito, assegurado pelo Estado. A especialidade de medicina geral e familiar foi também criada e implementada. Apesar da maioria da população ser utilizadora do SNS, há vários subsistemas de seguros de saúde, mas a população coberta por estes também tem acesso aos serviços do SNS, traduzindo-se, muitas vezes, numa dupla cobertura.

Sucessivas tentativas de reforma do SNS conduziram ao entendimento de que não foi dada a devida atenção ao acesso aos cuidados de saúde, sendo necessário projetar políticas de saúde que se traduzam em soluções técnicas que realmente funcionem. É igualmente importante que a base política de qualquer reforma seja alicerçada numa análise rigorosa das questões económicas, sociais, culturais e políticas (Observatóro Português dos Sistemas de Saúde [OPSS], 2008).

Em 2005 começou uma nova fase no sector da saúde, especialmente em relação aos CSP, focando em particular a reorganização dos centros de saúde, com a criação de Unidades de Saúde Familiar (USF), constituídas por equipas multidisciplinares com autonomia técnica e funcional, e de outras soluções 
mais adequadas para a prestação de cuidados de saúde primários. O impacto desta reforma está a ser acompanhado, o que justifica a necessidade de avaliar os seus resultados, em particular no que diz respeito às melhores práticas em CSP.

Do ponto de vista organizacional dos CSP, pode referir-se a existência, em 2005, de 351 centros de saúde, 1823 extensões de saúde e 254 Serviços de Atendimento Permanente (SAPs) em Portugal Continental, de acordo com o Instituto de Gestão Informática e Financeira da Saúde (2006). Em termos de modelo de contratualização, constatava-se a ausência de um papel claro e bem definido de um agente que desse resposta às necessidades dos diversos atores com responsabilidades no sector da saúde, especialmente no que diz respeito à negociação de objetivos e metas. De acordo com um estudo da Organização para a Cooperação e Desenvolvimento Económico (2006), os gastos com a saúde aumentariam no futuro, estimando-se representar 13.1\% do PIB em 2050, se não fossem implementadas reformas para contrariar esta tendência.

A prestação de serviços de saúde é caracterizada por um grande número de utilizadores sem acesso a médico de família, especialmente na área geográfica do litoral e nas grandes áreas urbanas. Os cidadãos podem escolher livremente o centro de saúde onde se inscrevem, bem como o seu profissional de medicina geral e familiar. Este médico fica designado como médico de família do utente para todas as consultas. No entanto, em 2005, cerca de $10.6 \%$ dos utentes do SNS não tinham médico de família (OPSS, 2007).

Durante todo o ano de 2007 houve um compromisso do Ministério da Saúde para a criação de mais USF e, a 31 de dezembro de 2007, já se encontravam 104 USF em funcionamento. ${ }^{1}$

Foram estabelecidos modelos de acompanhamento, avaliação e contratualização, com padrões de exigência elevados. Estes conduziram à criação de um clima de confiança entre os profissionais de saúde envolvidos e as Administrações Regionais de Saúde (ARS). O ambiente criado mobilizou cerca de 1300 médicos, 1300 enfermeiros e 950 funcionários administrativos, que aceitaram o desafio e se disponibilizaram para trabalhar em USF. Para além destas mudanças organizacionais, a lista de utentes inscritos, em média,

\footnotetext{
${ }^{1}$ Dados retirados do sítio www.mcsp.min-saude.pt, consultado em 15 de janeiro de 2012.
} 
aumentou para cerca de 1750 utentes por profissional de medicina geral e familiar (OPSS, 2008).

A 22 de fevereiro de 2008 foi publicado o Decreto-Lei n. ${ }^{\circ}$ 28/2008, que regulamenta a criação dos ACES. Estes são definidos como "serviços públicos de saúde com autonomia administrativa, constituídos por várias unidades funcionais, que agrupam um ou mais centros de saúde, e que têm por missão garantir a prestação de cuidados de saúde primários à população de determinada área geográfica". A constituição dos ACES pressupõe a sua organização em unidades funcionais, nomeadamente em USF, unidades de cuidados de saúde personalizados (UCSP - integram os restantes médicos de família e outros profissionais, mas não estão organizados em USF), unidades de cuidados na comunidade, unidades de saúde pública e unidades de recursos assistenciais partilhados. O ACES também prevê a existência de unidades de apoio à sua governação, como é o caso do Conselho da Comunidade, o Conselho Clínico, a Unidade de Apoio à Gestão e o Gabinete do Cidadão. Importa realçar que a autonomia do ACES é administrativa e está intimamente relacionada com a existência de um contrato-programa, a ser estabelecido entre o ACES e a ARS, Instituto Público.

De acordo com o Decreto-Lei n. ${ }^{\circ} 222 / 2007$, publicado em 29 de maio, as cinco ARS existentes "têm por missão garantir à população da respectiva área geográfica de intervenção o acesso à prestação de cuidados de saúde de qualidade, adequando os recursos disponíveis às necessidades em saúde e cumprir e fazer cumprir o Plano Nacional de Saúde na sua área de intervenção".

Em 2010, pela primeira vez, os Diretores Executivos/Conselhos Clínicos, com o apoio dos Departamentos de Contratualização das ARS, desencadearam a contratualização interna com as USF, já iniciada anteriormente, e o mesmo modelo foi adaptado para as UCSP.

Conforme consta no contrato-programa de 2010, para um futuro a curto prazo "espera-se que o processo de contratualização e a sofisticação da alocação dos recursos permita uma efetiva autonomização e responsabilização dos ACES pela efetividade dos cuidados de saúde prestados à população". 
4. Dados e modelos desenvolvidos atendendo às preferências do Grupo de Decisores

Esta secção descreve a avaliação de desempenho das 12 unidades prestadoras de cuidados de saúde monitorizadas pelo ACES Baixo Mondego I, com dados relativos a 2010. As unidades a avaliar são USF e UCSP, uma vez que todas elas estão no mesmo contrato-programa estabelecido entre o ACES e a ARS e prosseguem os mesmos objetivos. A avaliação de desempenho destas unidades é realizada periodicamente pelo ACES, tendo como base os indicadores que constam no Plano de Desempenho, sem atender a diferenças existentes a nível organizacional. Portanto, e a pedido do GD, manteve-se o mesmo critério na avaliação proposta neste estudo, podendo-se considerar que as unidades são homogéneas.

A monitorização e acompanhamento destas unidades assenta num conjunto de 15 indicadores agrupados em quatro grandes áreas: acesso, desempenho assistencial, qualidade percecionada e desempenho económico, cujas metas são negociadas entre a unidade e o Diretores Executivos/Conselhos Clínicos, tendo em consideração os objectivos de saúde estabelecidos pelo ACES e pela ARS. Um bom desempenho por parte das unidades poderá traduzir-se na atribuição de recompensas de vária ordem.

Os dados analisados foram extraídos de diversos sistemas de informação, desde os que são utilizados para monitorizar as atividades assistenciais até aos do departamento de contabilidade e gestão. A fim de incluir na análise um fator que reflete a qualidade percecionada de serviço, foi utilizada informação sobre a satisfação dos utentes com a qualidade do serviço, proveniente de um inquérito (Avaliação sobre o funcionamento das Unidades de Saúde do ACES, 2010). Este abrange 19 perguntas sobre acessibilidade aos serviços, informação e atendimento, instalações das unidades de saúde e dados sociodemográficos. O inquérito não considerou um indicador de satisfação global, mas foi entendido pelo GD que a pergunta "quando adoece de repente, consegue consulta no médico de família no próprio dia?" (resposta sim/não) seria uma boa aproximação da satisfação dos utentes com a qualidade do serviço.

Face ao objetivo de avaliação do desempenho das 12 DMUs (USF e UCSP) monitorizadas pelo ACES Baixo Mondego I, foi explicada ao GD a metodologia 
proposta por Gouveia et al. (2008). O GD aceitou esta abordagem que considerou muito útil, dada a possibilidade de usar múltiplos fatores num modelo integrado e a capacidade de identificar as melhores práticas, as fontes de ineficiência, as diferenças relativamente às melhores práticas, bem como as oportunidades de melhoria.

\subsection{Definição dos modelos}

Depois de traçados os objetivos do estudo, foram definidos os fatores de input e output para cada modelo, atendendo aos indicadores das principais áreas do Plano de Desempenho, que eram essencialmente rácios. Como resultado de reuniões surgiram vários modelos diferentes, pois vários entendimentos possíveis decorriam das diferentes perspectivas de avaliação utilizadas. No entanto, neste trabalho apenas serão apresentados dois desses modelos.

Com o objetivo de combinar desempenho assistencial, qualidade percecionada e desempenho económico, foi desenvolvido um modelo de eficiência face a custos. Neste, os custos associados à prestação de cuidados de saúde primários foram considerados como inputs; como outputs foram incluídos os fatores considerados como a principal atividade dos centros de saúde, juntamente com o fator relativo à satisfação dos utentes com a qualidade do serviço de saúde prestado. Os outputs (à exceção do último referido) são separados por tipo de consulta e por grupo etário dos utentes, a fim de controlar o "case-mix" (Tabela 1). Este modelo foi chamado de "eficiência dos serviços de saúde prestados atendendo às características do utente", a partir de agora referido como modelo 1.

Uma vez que no modelo 1 não está refletido o trabalho de enfermagem, houve a necessidade por parte do GD de criar um segundo modelo de eficiência (modelo 2). Este modelo, considerado mais abrangente, alia os vários eixos de avaliação mencionados no Plano de Desempenho, expressando em simultâneo todo o trabalho médico, de enfermagem e administrativo. Concordou-se com a inclusão de nove fatores para a construção deste modelo (ver Tabela 2), cujos inputs são os indicadores económicos do Plano de Desempenho, os primeiros quatro outputs estão relacionados com o acesso 
(número de utentes inscritos com pelo menos uma consulta no ano de referência) e desempenho assistencial e o último output é relativo à qualidade percecionada. Este modelo fornece ao GD uma perspetiva diferente das unidades em avaliação e foi considerado muito importante, uma vez que as recompensas institucionais são dependentes do desempenho das unidades em alguns destes fatores, nomeadamente o número de domicílios médicos e de enfermagem.

Tabela 1. Fatores do modelo 1.

\begin{tabular}{|c|c|}
\hline Inputs & Outputs \\
\hline $\begin{array}{l}\mathrm{x}_{\text {MCDT: }} \text { custo total com meios } \\
\text { complementares de diagnóstico e } \\
\text { tratamento faturados ao SNS }\end{array}$ & $\begin{array}{l}\text { Y }_{C P F} \text { : número de consultas médicas } \\
\text { de planeamento familiar }\end{array}$ \\
\hline $\begin{array}{l}\mathrm{x}_{M E D} \text { : custo total com medicamentos } \\
\text { faturados ao SNS }\end{array}$ & $\begin{array}{l}\text { Yсsм: número de consultas de saúde } \\
\text { materna }\end{array}$ \\
\hline $\mathrm{x}_{R H}$ : custo total com recursos humanos & $\begin{array}{l}\text { Y } \text { cSI: número de consultas de saúde } \\
\text { infantil (idade } 0-18 \text { ) }\end{array}$ \\
\hline \multirow[t]{3}{*}{$\begin{array}{l}\text { Xoc: custos totais com medicamentos, } \\
\text { materiais de consumo clínico e outros }\end{array}$} & $\begin{array}{l}\text { YcSA: número de consultas de saúde a } \\
\text { adultos (idade 19-64) }\end{array}$ \\
\hline & $\begin{array}{l}\text { Ycss: número de consultas de saúde a } \\
\text { seniores (idade superior ou igual a } \\
65 \text { ) }\end{array}$ \\
\hline & $\begin{array}{l}\text { YsQ: satisfação com a qualidade dos } \\
\text { serviços }\end{array}$ \\
\hline
\end{tabular}

Amplamente discutidos com o GD e em concordância com os indicadores estabelecidos no Plano de Desempenho, estes inputs e outputs são típicos deste tipo de aplicação DEA (e.g. Amado \& Santos, 2009).

\subsection{Construção das funções de utilidade dos fatores}

Existem várias técnicas para obter informações de preferência de um Grupo de Decisores/Decisor com o propósito de construir funções de utilidade compatíveis com as suas respostas (ver von Winterfeldt \& Edwards, 1986; Goodwin \& Wright 1998); no entanto, as perguntas para esse fim devem ser 
enquadradas no contexto de cada estudo. No método de Gouveia et al. (2008) o objetivo de converter os fatores numa escala de utilidade (funções de utilidade não-lineares) é o de espelhar as preferências do GD. Para construir as funções de utilidade seguiu-se um protocolo de diálogo já utilizado por Almeida e Dias (2012).

Tabela 2. Fatores do modelo 2.

\begin{tabular}{|c|c|}
\hline Inputs & Outputs \\
\hline $\begin{array}{l}\mathrm{x}_{M C D T}: \text { custo total com meios } \\
\text { complementares de diagnóstico e } \\
\text { tratamento faturados ao SNS }\end{array}$ & $\begin{array}{l}\mathrm{y}_{D M} \text { : número de domicúlios médicos } \\
\mathrm{y}_{C M} \text { : número de consultas médicas na }\end{array}$ \\
\hline $\begin{array}{l}\mathrm{x}_{M E D}: \text { custo total com medicamentos } \\
\text { faturados ao SNS }\end{array}$ & $\begin{array}{l}\mathrm{y}_{D E} \text { : número de domicílios de } \\
\text { enfermagem }\end{array}$ \\
\hline $\mathrm{x}_{R H}$ : custo total com recursos humanos & $\begin{array}{l}\text { y }_{C E}: \text { número de consultas de } \\
\text { enfermagem na unidade }\end{array}$ \\
\hline $\begin{array}{l}\mathrm{x}_{O C} \text { : custos totais com medicamentos, } \\
\text { materiais de consumo clínico e outros }\end{array}$ & $\begin{array}{l}\text { ysQ: satisfação com a qualidade dos } \\
\text { serviços }\end{array}$ \\
\hline
\end{tabular}

O protocolo de diálogo baseou-se na comparação entre o mérito de aumentar um output (ou diminuir um input) de $a$ para $b$ contra o aumento do mesmo output (ou diminuição do mesmo input) de $a^{\prime}$ para $b^{\prime}$, mantendo todos os outros níveis de desempenho iguais, e pedindo ao GD para ajustar um destes quatro valores de tal forma que o aumento de mérito seja aproximadamente igual. Para fazer esta conversão será apenas assumido que as funções são contínuas.

Por exemplo, considerando o output $\mathrm{y}_{D M}$ a questão colocada ao GD foi: "Tem mais mérito aumentar o número de domicílios médicos de 3 para 600 ou de 600 para 1200, mantendo todos os outros desempenhos iguais?" A resposta foi: "tem mais mérito aumentar de 600 para 1200". Então o nível foi ajustado e a questão foi reformulada como: "Tem mais mérito aumentar o número de domicílios médicos de 3 para 800 ou de 800 para 1200, mantendo todos os outros desempenhos iguais?" A resposta foi: "tem igual mérito". Um procedimento análogo foi utilizado para dicotomizar os intervalos de mérito $[3,800]$ e $[800,1200]$. 
As funções de utilidade, para a maioria dos fatores, foram representadas por aproximação a funções lineares definidas por ramos. Quando as respostas do GD se conseguiam ajustar a curvas pré-definidas (como funções logarítmicas) as funções utilidade foram representadas por essas funções conhecidas.

Os intervalos encontrados foram escolhidos com o intuito de incluir as gamas de desempenho observadas para cada fator, mais (ou menos) uma margem de tolerância máxima (neste caso de 20\%).

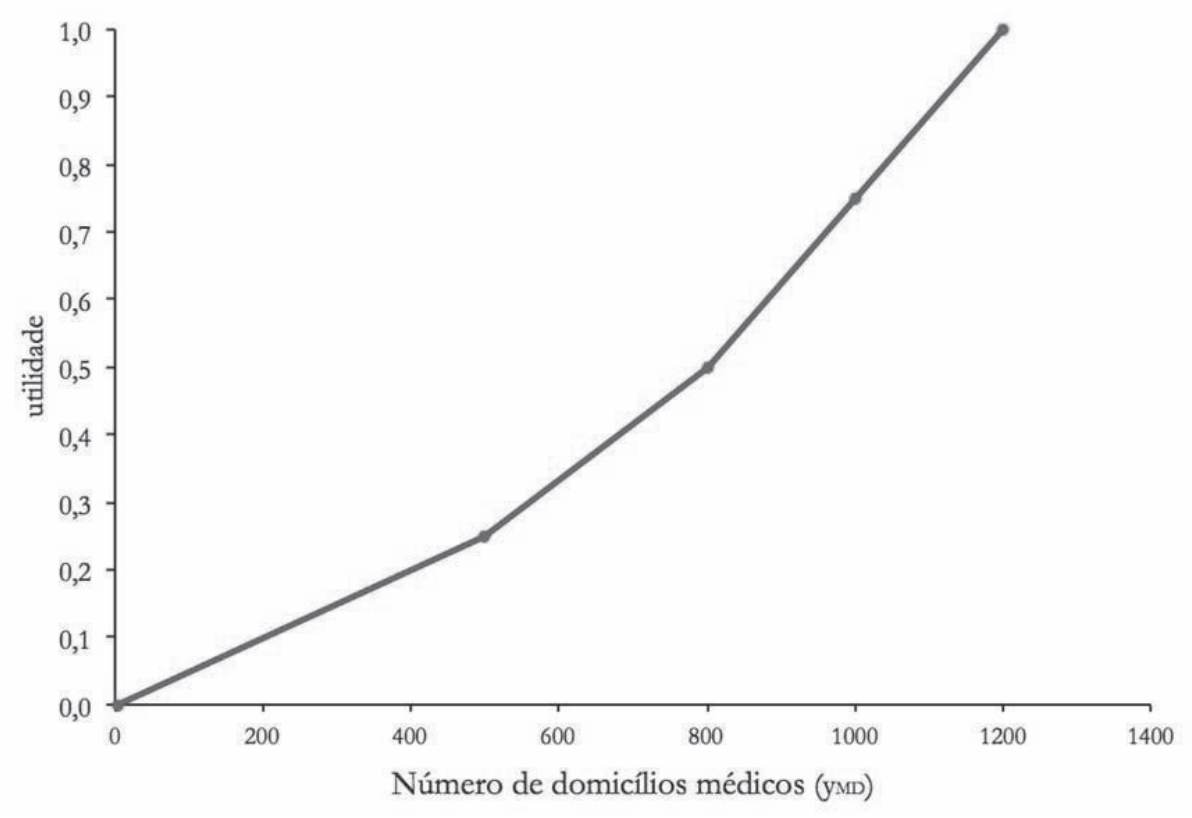



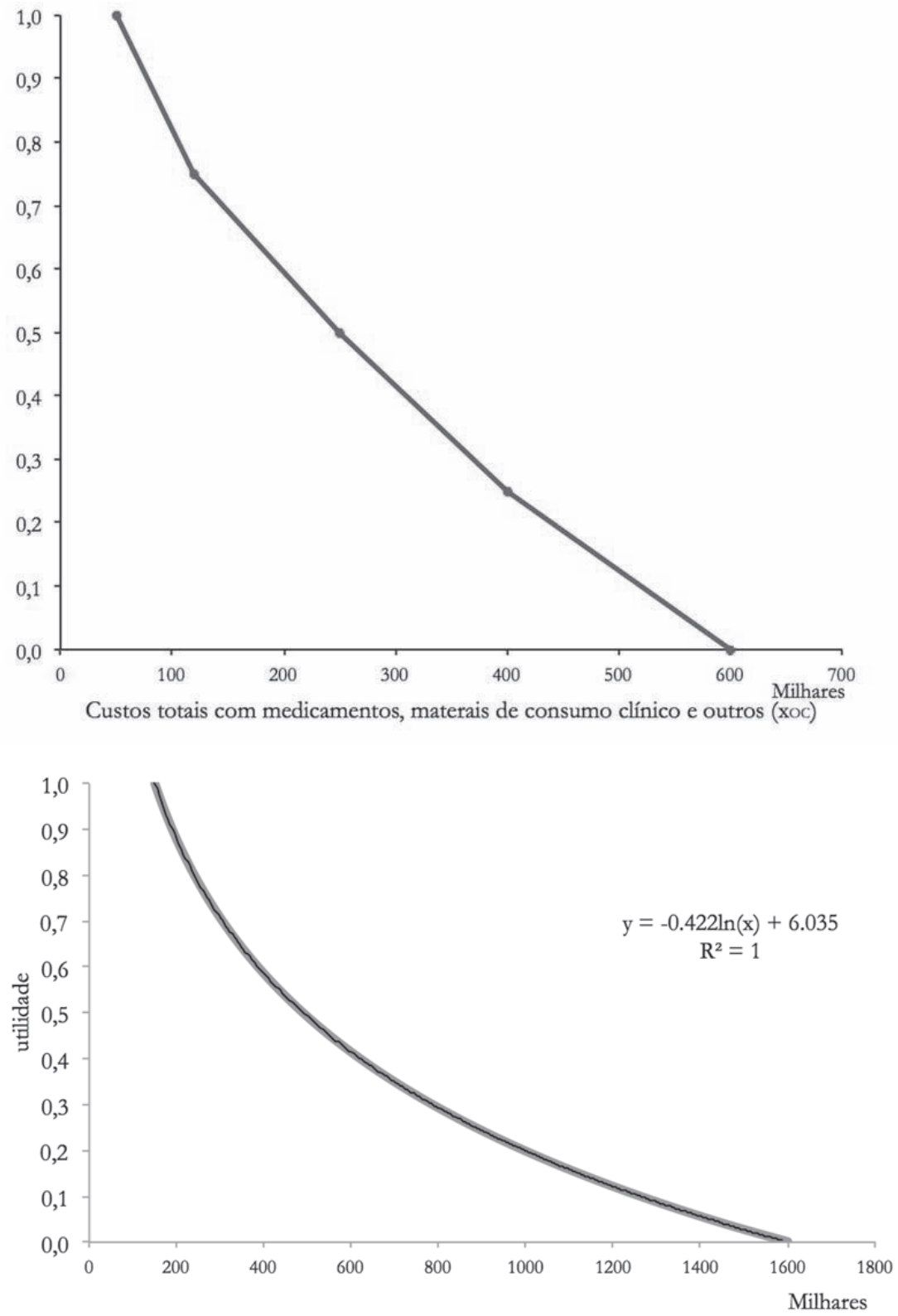

Custo total com meios complementares de diagnóstico e tratamento faturado ao SNS (хмCDT)

Figura 1. Três das funções de utilidade eliciadas. 
As funções de utilidade lineares definidas por ramos para alguns dos fatores estão exibidas na Figura 1. Por exemplo, para o número de domicílios médicos:

$u_{D M}(1200)-u_{D M}(1000)=u_{D M}(1000)-u_{D M}(800)=u_{D M}(800)-u_{D M}(500)=$ $=u_{D M}$ (500) $-u_{D M}$ (3), mantendo-se todos os outros níveis de desempenho iguais. As outras funções de utilidade para os restantes fatores output dos modelos ( $\left.\mathrm{y}_{C P F}, \mathrm{y}_{C S M}, \mathrm{y}_{C S I}, \mathrm{y}_{C S A}, \mathrm{y}_{C S S}, \mathrm{y}_{C M}, \mathrm{y}_{D E}, \mathrm{y}_{C E}, \mathrm{e} \mathrm{y}_{S Q}\right)$ são semelhantes à função de utilidade $\mathrm{y}_{D M}$.

Para os custos $\mathrm{x}_{M C D T}, \mathrm{x}_{M E D}$, e $\mathrm{x}_{R H}$, as funções de utilidade foram obtidas realizando o ajustamento de uma função conhecida às preferências reveladas pelo GD.

\subsection{Restrições aos coeficientes de ponderação}

Para os modelos apresentados houve a necessidade de incorporar restrições aos coeficientes de ponderação porque, caso contrário, não era possível alcançar uma classificação das unidades com discriminação aceitável (veja-se Dyson et al., 2001). Isto foi explicado ao GD, acrescentando que a introdução de restrições aos coeficientes de ponderação nos modelos traduz melhor os objetivos da organização e, portanto, garante resultados significativamente mais próximos daquilo que o GD considera como sendo as melhores práticas.

Existem na literatura várias abordagens para a definição de restrições aos coeficientes de ponderação, podendo a especificação destes de forma adequada revelar-se um desafio (Salo \& Hämäläinen, 2001; Podinovski, 2004).

A técnica de swings começa pela construção de duas hipóteses extremas, B e G; a primeira diz respeito ao pior desempenho (utilidade 0) em todos os critérios e a segunda corresponde ao melhor desempenho (utilidade 1). O protocolo de diálogo consiste em consultar o decisor no sentido deste olhar para potenciais ganhos ao passar de B para G em cada critério e, em seguida, decidir quais os critérios que prefere mudar para a hipótese G. Supõe-se que a transição da hipótese B para a hipótese G, num determinado critério, vale 100 unidades numa escala hipotética. Em seguida, é solicitada ao decisor a atribuição de um valor $(<100)$ ao segundo critério movido para G, depois ao 
terceiro critério e assim sucessivamente, até que o último critério é movido para G.

O procedimento utilizado neste trabalho foi: primeiro obter uma ordenação de coeficientes de ponderação (baseado na técnica de swings) e, depois, estabelecer um limite para a razão entre os coeficientes de ponderação que ficaram em primeiro e em último lugar (pergunta de trade-off), por forma a evitar pesos nulos.

Ao incluir os coeficientes de ponderação, a Fase 1 e a Fase 2 (ver secção 2) passam a assumir outras formulações: às restrições do programa linear da Fase 1 acrescentam-se as restrições aos pesos, ao passo que no programa linear da Fase 2 é necessário permitir às folgas $s_{c}$ ter valores negativos, retirando-se a restrição $s_{c} \geq 0$ (ver detalhes em Almeida \& Dias, 2012).

\section{Resultados}

A implementação computacional do método, bem como das suas extensões, foram realizadas recorrendo a uma folha de cálculo.

O analista em conjunto com o GD construíram dois modelos (1 e 2) para avaliar a eficiência da unidades de CSP. A Tabela 3 apresenta os dados originais e as utilidades correspondentes para o modelo 1. 
Tabela 3. Desempenhos das DMUs nas escalas originais e na escala de utilidades para o modelo 1.

\begin{tabular}{|c|c|c|c|c|c|c|c|c|c|c|}
\hline & \multicolumn{10}{|c|}{ Fatores nas escalas originais } \\
\hline DMU & $\mathrm{X} M C D T$ & $\mathrm{X}_{M E D}$ & $\mathrm{X}_{R H}$ & Xoc & YCPF & $\mathrm{y} \operatorname{CsM}$ & $\mathrm{y} C S I$ & $\mathrm{y} \operatorname{CSA}$ & $\mathrm{y} \operatorname{css}$ & $\mathrm{y} s Q$ \\
\hline 1 & 362900 & 1114935 & 1398033 & 123382 & 845 & 323 & 5133 & 25380 & 17149 & 92 \\
\hline 2 & 463549 & 1192330 & 1050558 & 128606 & 449 & 311 & 3197 & 19983 & 14783 & 74 \\
\hline 3 & 466561 & 1113734 & 1589818 & 101750 & 85 & 76 & 691 & 5136 & 2434 & 81 \\
\hline 4 & 277140 & 1321779 & 1001297 & 152974 & 1628 & 605 & 4934 & 19283 & 13811 & 83 \\
\hline 5 & 232565 & 1147295 & 1101200 & 141864 & 732 & 363 & 1668 & 17775 & 12108 & 62 \\
\hline 6 & 613535 & 2491923 & 1724028 & 218890 & 2534 & 782 & 5068 & 29422 & 18854 & 75 \\
\hline 7 & 1332317 & 4365605 & 2368401 & 360011 & 3726 & 1081 & 8073 & 54200 & 40307 & 77 \\
\hline 8 & 701121 & 2289334 & 2225321 & 491140 & 2641 & 1142 & 4530 & 41247 & 27858 & 80 \\
\hline 9 & 314090 & 1196353 & 1054564 & 65416 & 666 & 502 & 4513 & 21909 & 13476 & 91 \\
\hline 10 & 606148 & 2954054 & 1348651 & 203098 & 3353 & 662 & 5068 & 29422 & 18854 & 54 \\
\hline 11 & 1007909 & 3350321 & 1933791 & 371468 & 4763 & 1084 & 6587 & 42838 & 30037 & 78 \\
\hline 12 & 708936 & 2793686 & 2227392 & 278936 & 3087 & 1052 & 5328 & 35475 & 25593 & 74 \\
\hline \multicolumn{11}{|c|}{ Fatores em escalas de utilidade } \\
\hline DMU & $u_{M C D T}$ & $\mathcal{U}_{M E D}$ & $u_{R H}$ & $\mathcal{U} O C$ & $\mathcal{U}_{C P F}$ & $\mathcal{U}_{C S M}$ & $\mathcal{U}_{C S I}$ & $\mathcal{U}_{C S A}$ & $u_{C S S}$ & $u_{S Q}$ \\
\hline 1 & 0.633 & 0.824 & 0.572 & 0.743 & 0.084 & 0.123 & 0.392 & 0.256 & 0.210 & 0.750 \\
\hline 2 & 0.529 & 0.789 & 0.788 & 0.733 & 0.042 & 0.117 & 0.193 & 0.190 & 0.178 & 0.400 \\
\hline 3 & 0.527 & 0.824 & 0.475 & 0.815 & 0.003 & 0.010 & 0.014 & 0.014 & 0.006 & 0.521 \\
\hline 4 & 0.746 & 0.735 & 0.825 & 0.687 & 0.168 & 0.253 & 0.367 & 0.182 & 0.164 & 0.563 \\
\hline 5 & 0.820 & 0.809 & 0.753 & 0.708 & 0.072 & 0.141 & 0.083 & 0.164 & 0.140 & 0.220 \\
\hline 6 & 0.411 & 0.406 & 0.413 & 0.560 & 0.271 & 0.364 & 0.384 & 0.324 & 0.234 & 0.417 \\
\hline 7 & 0.084 & 0.115 & 0.173 & 0.317 & 0.457 & 0.568 & 0.759 & 0.737 & 0.633 & 0.450 \\
\hline 8 & 0.355 & 0.450 & 0.220 & 0.136 & 0.288 & 0.618 & 0.316 & 0.521 & 0.381 & 0.500 \\
\hline 9 & 0.694 & 0.787 & 0.785 & 0.945 & 0.065 & 0.205 & 0.314 & 0.213 & 0.159 & 0.729 \\
\hline 10 & 0.416 & 0.318 & 0.599 & 0.590 & 0.399 & 0.289 & 0.384 & 0.324 & 0.234 & 0.140 \\
\hline 11 & 0.202 & 0.253 & 0.326 & 0.298 & 0.659 & 0.570 & 0.573 & 0.547 & 0.417 & 0.467 \\
\hline 12 & 0.350 & 0.347 & 0.219 & 0.452 & 0.357 & 0.543 & 0.416 & 0.425 & 0.343 & 0.400 \\
\hline
\end{tabular}

Como anteriormente foi referido, para estes dois modelos, dada a relação entre o número de fatores e o número de unidades em avaliação, foi necessário incorporar restrições aos coeficientes de ponderação. 
De acordo com o procedimento explicado na sub-secção 4.3, as restrições aos coeficientes de ponderação foram obtidas pedindo ao GD para comparar os "swings" da utilidade de 0 para 1 para o modelo 1 , como descrito na Tabela 4.

O GD foi convidado a considerar uma unidade com o desempenho no nível 0 para todos os fatores e perguntou-se: "se pudesse melhorar um e apenas um fator para o nível 1, qual seria?". A resposta do GD foi unânime: "custo total com medicamentos faturados ao SNS". Isto permite a ilação de que $w_{\text {MED }}$ é o maior coeficiente de escala. Ao repetir esta pergunta sucessivamente para os demais fatores, e depois de alguma discussão, o GD confluiu na seguinte ordenação para os coeficientes de ponderação:

$$
w_{\mathrm{MED}} \geq w_{\mathrm{MCDT}} \geq w_{\mathrm{RH}} \geq w_{\mathrm{SQ}} \geq w_{\mathrm{CPF}} \geq w_{\mathrm{CSI}} \geq w_{\mathrm{CSM}} \geq w_{\mathrm{OC}} \geq w_{\mathrm{CSA}} \geq w_{\mathrm{CSS}} .
$$

Tabela 4. Desempenhos extremos associados com os níveis de utilidade 0 e 1 (modelo 1).

\begin{tabular}{lllllllllll}
\hline $\begin{array}{c}\text { Nível de } \\
\text { utilidade }\end{array}$ & $\mathrm{x}_{M C D T}$ & $\mathrm{x}_{M E D}$ & $\mathrm{x}_{R H}$ & $\mathrm{x} O C$ & $\mathrm{y}_{C P F}$ & $\mathrm{y}_{C S M}$ & $\mathrm{y}_{C S I}$ & $\mathrm{y}_{C S A}$ & $\mathrm{y}_{C S S}$ & $\mathrm{y}_{S Q}$ \\
\hline$u()=0$. & 1600000 & 5500000 & 3000000 & 600000 & 60 & 55 & 500 & 4000 & 2000 & 40 \\
$u()=1$. & 150000 & 800000 & 800000 & 50000 & 6000 & 1500 & 10000 & 65000 & 50000 & 100 \\
\hline
\end{tabular}

Com o objetivo de evitar valores nulos nos coeficientes de ponderação, foi feita ao GD uma pergunta de trade-off para limitar a relação dos coeficientes de ponderação classificados na primeira $\left(w_{\text {MED }}\right)$ e última posição $\left(w_{\text {CSS }}\right)$ : "Qual seria o maior valor $z$ em euros correspondente ao custo total com medicamentos faturados ao SNS que permitiria a uma unidade com 50000 consultas de saúde a seniores realizadas e custo total com medicamentos faturados ao SNS associado de 5500000 euros ser considerada como tendo mais mérito do que uma unidade com 2000 consultas de saúde a seniores realizadas e com um custo total com medicamentos faturados ao SNS de $z$ euros?". A resposta permite construir a seguinte desigualdade:

$$
w_{\mathrm{CSS}} u(50000)+w_{\mathrm{MED}} u(5500000) \geq w_{\mathrm{CSS}} u(2000)+w_{\mathrm{MED}} u(z)
$$

O limite obtido foi $z=2500000$. Com o aumento deste valor o GD começava a hesitar se a desigualdade se manteria. Substituindo esse valor de $z$ na expressão anterior: $w_{\mathrm{MED}} \leq 2.47 w_{\mathrm{CSS}}$.

Os resultados para o modelo 2 estão representados nas Tabela 5 e 6. 
Tabela 5. Resultados da Fase 1, com restrições aos coeficientes de ponderação (modelo 1).

\begin{tabular}{|c|c|c|c|c|c|c|c|c|c|c|c|}
\hline & \multicolumn{11}{|c|}{ Fase 1} \\
\hline DMU & $d^{*}$ & $w_{M C D T}^{*}$ & $w_{M E D}^{*}$ & $w_{R H}^{*}$ & $w_{O C}^{*}$ & $w_{C P F}^{*}$ & $w^{*} C S M$ & $w_{C S I}^{*}$ & $w_{C S A}^{*}$ & $w_{C S S}^{*}$ & $w^{*} S_{Q}$ \\
\hline 1 & 0.0223 & 0.087 & 0.215 & 0.087 & 0.087 & 0.087 & 0.087 & 0.087 & 0.087 & 0.087 & 0.087 \\
\hline 2 & 0.0813 & 0.171 & 0.171 & 0.171 & 0.069 & 0.069 & 0.069 & 0.069 & 0.069 & 0.069 & 0.069 \\
\hline 3 & 0.1426 & 0.087 & 0.215 & 0.087 & 0.087 & 0.087 & 0.087 & 0.087 & 0.087 & 0.087 & 0.087 \\
\hline 4 & 0.0047 & 0.122 & 0.122 & 0.122 & 0.049 & 0.122 & 0.122 & 0.122 & 0.049 & 0.049 & 0.122 \\
\hline 5 & 0.0566 & 0.171 & 0.171 & 0.171 & 0.069 & 0.069 & 0.069 & 0.069 & 0.069 & 0.069 & 0.069 \\
\hline 6 & 0.1114 & 0.100 & 0.100 & 0.100 & 0.100 & 0.100 & 0.100 & 0.100 & 0.100 & 0.100 & 0.100 \\
\hline 7 & 0.0605 & 0.100 & 0.100 & 0.100 & 0.100 & 0.100 & 0.100 & 0.100 & 0.100 & 0.100 & 0.100 \\
\hline 8 & 0.1112 & 0.100 & 0.100 & 0.100 & 0.100 & 0.100 & 0.100 & 0.100 & 0.100 & 0.100 & 0.100 \\
\hline 9 & -0.0248 & 0.156 & 0.156 & 0.156 & 0.063 & 0.063 & 0.063 & 0.063 & 0.063 & 0.063 & 0.156 \\
\hline 10 & 0.1204 & 0.100 & 0.100 & 0.100 & 0.100 & 0.100 & 0.100 & 0.100 & 0.100 & 0.100 & 0.100 \\
\hline 11 & 0.0585 & 0.100 & 0.100 & 0.100 & 0.100 & 0.100 & 0.100 & 0.100 & 0.100 & 0.100 & 0.100 \\
\hline 12 & 0.1044 & 0.100 & 0.100 & 0.100 & 0.100 & 0.100 & 0.100 & 0.100 & 0.100 & 0.100 & 0.100 \\
\hline
\end{tabular}

Tabela 6. Resultados da Fase 2, com restrições aos coeficientes de ponderação e folgas livres (modelo 1).

\begin{tabular}{|c|c|c|c|c|c|c|c|c|c|c|}
\hline \multirow[b]{2}{*}{ DMU } & \multicolumn{10}{|c|}{ Fase 2} \\
\hline & $s_{M C D T}^{*}$ & $s_{M E D}^{*}$ & $s_{R H}^{*}$ & $s^{*}{ }_{O C}$ & $s_{C P F}^{*}$ & $s_{C S M}^{*}$ & $s_{C S I}^{*}$ & $s_{C S A}^{*}$ & $s_{C S S}^{*}$ & $s_{S Q}^{*}$ \\
\hline 1 & 0.061 & -0.037 & 0.213 & 0.201 & -0.019 & 0.082 & -0.078 & -0.043 & -0.051 & -0.021 \\
\hline 2 & 0.164 & -0.002 & -0.003 & 0.211 & 0.023 & 0.088 & 0.121 & 0.023 & -0.018 & 0.329 \\
\hline 3 & 0.167 & -0.037 & 0.311 & 0.130 & 0.062 & 0.195 & 0.300 & 0.200 & 0.153 & 0.208 \\
\hline 4 & -0.053 & 0.052 & -0.039 & 0.258 & -0.103 & -0.048 & -0.053 & 0.031 & -0.005 & 0.167 \\
\hline 5 & -0.127 & -0.022 & 0.033 & 0.237 & -0.007 & 0.064 & 0.231 & 0.049 & 0.019 & 0.509 \\
\hline 6 & 0.283 & 0.381 & 0.372 & 0.385 & -0.206 & -0.159 & -0.069 & -0.110 & -0.075 & 0313 \\
\hline 7 & 0.610 & 0.672 & 0.612 & 0.628 & -0.392 & -0.362 & -0.445 & -0.523 & -0.473 & 0.279 \\
\hline 8 & 0.339 & 0.337 & 0.565 & 0.809 & -0.223 & -0.413 & -0.002 & -0.308 & -0.222 & 0.229 \\
\hline 9 & & & & & & & & & & \\
\hline 10 & 0.277 & 0.469 & 0.186 & 0.355 & -0.334 & -0.084 & -0.069 & -0.110 & -0.075 & 0589 \\
\hline 11 & 0.492 & 0.534 & 0.459 & 0.647 & -0.594 & -0.365 & -0.259 & -0.334 & -0.258 & 0.263 \\
\hline 12 & 0.344 & 0.440 & 0.566 & 0.493 & -0.293 & -0.338 & -0.102 & -0.211 & -0.184 & 0.329 \\
\hline
\end{tabular}


Neste caso há apenas uma unidade eficiente (DMU 9), que é a referência para todos as outras (Tabela 5). Esta unidade tem as melhores práticas de acordo com as preferências do GD.

Obteve-se a seguinte ordenação:

DMU $9>$ DMU $4>$ DMU $1>$ DMU $5>$ DMU $11>$ DMU $7>$ DMU $2>$ DMU $12>$ DMU $8>$ DMU $6>$ DMU $10>$ DMU 3 .

Ao GD agradou que a DMU 9 ficasse em primeiro lugar mas ficou um pouco surpreendido, pois esperava que outra unidade assumisse o primeiro lugar. Este resultado suscitou o debate no GD, tendo surgido como possível explicação o facto de os fatores de output traduzirem pouco o trabalho de enfermagem. A DMU que ficou em último lugar foi realmente surpreendente, mas o GD conjeturou que existe uma forte possibilidade de não terem sido registadas todas as consultas médicas naquela unidade. No entanto, seria necessário averiguar melhor a causa desta última posição neste modelo.

O modelo 2 é aquele que o GD considera como sendo o mais abrangente, dado que combina os vários eixos de avaliação correspondentes ao acesso, desempenho assistencial, qualidade percecionada e desempenho económico e reflete todo o trabalho médico, de enfermagem e administrativo. Os dados originais e as utilidades correspondentes para o modelo 2 são expostos na Tabela 7

As restrições aos coeficientes de ponderação foram obtidas de forma semelhante às do modelo 1 e ao GD foi pedido para comparar os "swings" da utilidade de 0 para 1 apresentados na Tabela 8 .

A ordenação dos coeficientes de escala obtida foi:

$w_{M E D} \geq w_{M C D T} \geq w_{R H} \geq w_{D M} \geq w_{O C} \geq w_{S Q} \geq w_{C E} \geq w_{C M} \geq w_{D E}$.

De acordo com a resposta conseguida à questão de julgamento de indiferença, foi estabelecida a seguinte desigualdade: $w_{\mathrm{MED}} \leq 2.47 w_{\mathrm{DE}}$.

A ordenação das unidades é ( $d *$ mostrado entre parênteses):

DMU $1(-0.0562)>$ DMU $9(-0.0013)>$ DMU $4(0.0229)>$ DMU $5(0.0503)$ $>$ DMU $2(0.0725)>$ DMU $3(0.1395)>$ DMU $6(0.1668)>$ DMU 10 (0.1810) $>$ DMU $11(0.1886)>$ DMU $12(0.2003)>$ DMU 7 (0.2429) > DMU 8 (0.2619).

Embora a DMU 9 não esteja na primeira posição, continua a ser eficiente neste modelo. 
Tabela 7. Desempenhos das DMUs nas escalas originais e na escala de utilidades para o modelo 2 .

\begin{tabular}{|c|c|c|c|c|c|c|c|c|c|}
\hline \multirow[b]{2}{*}{ DMU } & \multicolumn{9}{|c|}{ Fatores nas escalas originais } \\
\hline & $\mathrm{X}_{M C D T}$ & $\mathrm{X}_{M E D}$ & $\mathrm{X}_{R H}$ & $\mathrm{X} O C$ & $\mathrm{y}_{D M}$ & $\mathrm{y}_{C M}$ & $\mathrm{y}_{D E}$ & $\mathrm{y}_{C E}$ & $\mathrm{y} s Q$ \\
\hline 1 & 362900 & 1114935 & 1398033 & 123382 & 985 & 48038 & 2006 & 24273 & 92 \\
\hline 2 & 463549 & 1192330 & 1050558 & 128606 & 241 & 37977 & 1424 & 20580 & 74 \\
\hline 3 & 466561 & 1113734 & 1589818 & 101750 & 96 & 8265 & 844 & 11677 & 81 \\
\hline 4 & 277140 & 1321779 & 1001297 & 152974 & 308 & 38102 & 1979 & 17150 & 83 \\
\hline 5 & 232565 & 1147295 & 1101200 & 141864 & 4 & 21665 & 792 & 27328 & 62 \\
\hline 6 & 613535 & 2491923 & 1724028 & 218890 & 195 & 50768 & 3645 & 34610 & 75 \\
\hline 7 & 1332317 & 4365605 & 2368401 & 360011 & 570 & 96530 & 4166 & 23781 & 77 \\
\hline 8 & 701121 & 2289334 & 2225321 & 491140 & 182 & 68599 & 987 & 34052 & 80 \\
\hline 9 & 314090 & 1196353 & 1054564 & 65416 & 302 & 40283 & 1756 & 17435 & 91 \\
\hline 10 & 606148 & 2954054 & 1348651 & 203098 & 244 & 45819 & 2249 & 45119 & 54 \\
\hline 11 & 1007909 & 3350321 & 1933791 & 371468 & 365 & 75904 & 5031 & 35040 & 78 \\
\hline \multirow[t]{2}{*}{12} & 70936 & 2793686 & 2227392 & 278936 & 246 & 64165 & 3878 & 34831 & 74 \\
\hline & \multicolumn{9}{|c|}{ Fatores em escalas de utilidade } \\
\hline DMU & $u_{M C D T}$ & $u_{M E D}$ & $u_{R H}$ & $u_{O C}$ & $u_{D M}$ & $u_{C M}$ & $u_{D E}$ & $u_{C E}$ & $u_{S Q}$ \\
\hline 1 & 0.633 & 0.824 & 0.572 & 0.743 & 0.731 & 0.194 & 0.185 & 0.225 & 0.750 \\
\hline 2 & 0.529 & 0.789 & 0.788 & 0.733 & 0.120 & 0.147 & 0.108 & 0.170 & 0.400 \\
\hline 3 & 0.527 & 0.824 & 0.475 & 0.815 & 0.047 & 0.008 & 0.032 & 0.039 & 0.521 \\
\hline 4 & 0.746 & 0.735 & 0.825 & 0.687 & 0.153 & 0.148 & 0.181 & 0.120 & 0.563 \\
\hline 5 & 0.820 & 0.809 & 0.753 & 0.708 & 0.001 & 0.071 & 0.025 & 0.274 & 0.220 \\
\hline 6 & 0.411 & 0.406 & 0.413 & 0.560 & 0.097 & 0.207 & 0.441 & 0.404 & 0.417 \\
\hline 7 & 0.084 & 0.115 & 0.173 & 0.317 & 0.308 & 0.478 & 0.528 & 0.217 & 0.450 \\
\hline 8 & 0.355 & 0.450 & 0.220 & 0.136 & 0.090 & 0.304 & 0.051 & 0.394 & 0.500 \\
\hline 9 & 0.694 & 0.787 & 0.785 & 0.945 & 0.150 & 0.158 & 0.152 & 0.124 & 0.729 \\
\hline 10 & 0.416 & 0.318 & 0.599 & 0.590 & 0.121 & 0.184 & 0.217 & 0.642 & 0.140 \\
\hline 11 & 0.202 & 0.253 & 0.326 & 0.298 & 0.182 & 0.349 & 0.672 & 0.411 & 0.467 \\
\hline 12 & 0.350 & 0.347 & 0.219 & 0.452 & 0.122 & 0.276 & 0.480 & 0.408 & 0.400 \\
\hline
\end{tabular}


Tabela 8. Desempenhos extremos associados com os níveis de utilidade 0 e 1 (modelo 2).

\begin{tabular}{|c|c|c|c|c|c|c|c|c|c|}
\hline $\begin{array}{l}\text { Nível de } \\
\text { utilidade }\end{array}$ & $\mathrm{X}_{M C D T}$ & $\mathrm{X}_{M E D}$ & $\mathrm{X}_{R H}$ & $\mathrm{X} O C$ & $\mathrm{y}_{D M}$ & $\mathrm{y}_{C M}$ & $\mathrm{y}_{D E}$ & $\mathrm{y}_{C E}$ & $\mathrm{y} s Q$ \\
\hline$u()=0$. & 1600000 & 5500000 & 3000000 & 600000 & 3 & 6500 & 600 & 9000 & 40 \\
\hline$u()=1$. & 150000 & 800000 & 800000 & 50000 & 1200 & 150000 & 6500 & 55000 & 100 \\
\hline
\end{tabular}

Os resultados alcançados corresponderam às expectativas do GD, em vários aspectos: a DMU 3 recupera, subindo várias posições no ranking, com a inclusão do trabalho de enfermagem; a DMU 5 está na $2 .^{a}$ posição entre as ineficientes, mantendo a $4 .^{a}$ posição no ranking geral; a DMU 1 é, como esperado, a melhor de todas as unidades, sendo uma referência para a grande maioria das unidades ineficientes (exceto para uma); a DMU 8 é classificada como a mais ineficiente, facto nada surpreendente para o GD.

\section{Melhorias das unidades ineficientes e outros desenvolvimentos}

Considere-se que $W$ denota o conjunto de vetores de coeficientes de ponderação compatíveis com o ranking e limite obtidos (ver 4.3.). A Fase 2 do método usa o vetor de ponderação ótimo em $W^{*}$ para resolver o modelo de PL da Fase 2 com as variáveis folga a assumir qualquer valor real. A solução é uma proposta de um alvo de eficiência (projeção) para cada DMU ineficiente. Para atingir o estado de eficiência, essas DMUs ineficientes devem mudar a sua utilidade em cada fator pelo valor indicado por $s^{*}$, considerando os dois modelos.

À partida, não havia restrições aos alvos: qualquer alvo era admitido desde que permitisse à DMU anular o seu défice de valor global. No entanto, tal não impedia que o alvo escolhido fosse pior nalguns fatores, desde que estas variações fossem suficientemente compensadas por melhorias nos restantes fatores. Logo, nem todas as mudanças propostas correspondem a melhorias em todos os fatores, uma vez que algumas folgas podiam ser negativas. Note-se, porém, que uma folga negativa para um fator de input corresponde a uma sugestão de aumentar o consumo desse input, e uma folga para um fator de output corresponde a uma sugestão de reduzir a produção desse output. O GD não concordou com estas possibilidades, uma vez que não tinha vantagem 
alguma em aumentar recursos, nomeadamente custos, nem em diminuir serviços prestados, pelo que houve a necessidade de propor uma outra solução. Considera-se que $u_{k}^{*}$ é a utilidade da melhor DMU usando o vetor de ponderação no conjunto ótimo $W^{*}$, ou seja, o valor da utilidade que a DMU $k$ deve alcançar:

$$
u_{k}^{*}=\sum_{c=1}^{q} w_{c}^{*} u_{c}\left(D M U_{k}\right)+d_{k}^{*}
$$

Sabe-se que o modelo de PL resolvido na Fase 2 do método de Gouveia et al. (2008) admite alvos ótimos alternativos, cada um dos quais corresponde a uma forma diferente de anular o valor da diferença $d_{k}^{*}$. Esses alvos correspondem a diferentes projeções sobre a fronteira eficiente. No presente caso, o GD tem a intenção de restringir os alvos de eficiência que se propõem alcançar $u_{k}^{*}$, não só para evitar os alvos que implicam um aumento de inputs ou uma redução dos outputs, mas também para escolher, dadas as características de cada unidade, quais os fatores que podem ser alterados.

O conjunto $S_{<}=\left\{c \in\{1, \ldots, q\}: s_{c}^{*}<0\right.$ na Fase 2$\}$ denota as folgas negativas no ótimo de (5); essas folgas vão passar agora a ser constantes nulas. O conjunto $S_{\geq}=\left\{c \in\{1, \ldots, q\}: S_{c}^{*} \geq 0\right.$ na Fase 2$\}$ denota as restantes folgas, que serão consideradas como variáveis não-negativas. Portanto, a formulação que conduz a um alvo alternativo que mantém ou melhora o desempenho de todos os fatores pode ser obtida resolvendo o modelo de PL (7), no qual a folga máxima (em termos de valor) deve ser minimizada para atingir o valor global de utilidade do alvo. Não são permitidas folgas negativas, mas o alvo já não será uma combinação convexa das DMUs observadas (para mais detalhes ver Almeida \& Dias, 2012).

A formulação abaixo difere da utilizada por Almeida e Dias (2012) na medida em que introduz um novo parâmetro $\sigma_{C}$ (um valor positivo) para limitar o valor que uma folga pode ter. O objetivo é evitar a fixação de alvos irrealistas, permitindo que as propostas de melhoria sejam mais ajustadas às exigências do GD. Os alvos nunca excedem o valor 1 em qualquer fator, devido à restrição $u_{c}\left(D M U_{k}\right)+s_{c} \leq 1$. Tal garante que a função de utilidade não ultrapassa os intervalos dos desempenhos obtidos. 


$$
\begin{array}{ll}
\min _{\rho_{k}, s} \rho_{k} \\
\text { s.a: } & \sum_{c \in S_{\geq}} w_{c}^{*} s_{c}=d_{k}^{*} \\
& u_{c}\left(D M U_{k}\right)+s_{c} \leq 1, c=1, \ldots, q \\
& s_{c}-\sigma_{c} \leq 0, c \in S_{\geq} \\
& \rho_{c} \geq s_{c} \geq 0, c=1, \ldots, q
\end{array}
$$

No que diz respeito ao modelo 1, todas as unidades ineficientes escolheram como par a DMU 9 e o GD começou por analisar os resultados em termos de propostas de melhoria das unidades ineficientes, bloqueando alterações nos fatores que tinham folgas negativas na Fase 2 (ver proposta 1 na Tabela 9), não permitindo que, nestes fatores, o alvo tivesse pior desempenho que o desempenho original das unidades.

Para algumas unidades a proposta 1 tem desempenhos atingíveis, mas para outras tais seriam impossíveis de alcançar. Esta realidade obrigou à construção de uma nova formulação, (8), na qual foi feita uma distribuição por todos os fatores (inputs e outputs) do valor da diferença relativamente ao par, de uma forma equilibrada, através do seguinte modelo de PL:

$$
\begin{array}{ll}
\min _{\rho_{k}, s} \rho_{k} \\
\text { s.a: } & \sum_{c=1}^{q} w_{c}^{*} s_{c}=d_{k}^{*} \\
& u_{c}\left(D M U_{k}\right)+s_{c} \leq 1, c=1, \ldots, q \\
& s_{c}-\sigma_{c} \leq 0, c=1, \ldots, q \\
& \rho_{c} \geq s_{c} \geq 0, c=1, \ldots, q
\end{array}
$$

Mesmo assim, o GD não entendeu como praticáveis, dadas as características de certas unidades, algumas das reduções propostas, nomeadamente no custo total com recursos humanos proposto para a DMU 3, e nos aumentos de algumas consultas propostas para as DMUs 6 e 10 (ver proposta 2 na Tabela 9). Neste último caso a justificação está relacionada com estatísticas nacionais associadas à taxa de natalidade, bem como ao facto de alguns utentes (apesar de inscritos no serviço de saúde público) preferirem ir ao serviço de saúde privado, possivelmente por motivos de desempenho percebido. Assim, surgiu a proposta de limitar a redução ou aumento de alguns fatores de input ou output, para as unidades 3, 6 e 10 no modelo 1. Para este efeito, a diferença entre a unidade em avaliação e a DMU 9 deveria ser suprimida pela redução ou aumento de outros fatores de input ou output (ver proposta 3 na Tabela 9). 
Tabela 9. Propostas de melhoria para as unidades ineficientes nas escalas originais (modelo 1)

\begin{tabular}{|c|c|c|c|c|c|c|c|c|c|c|}
\hline & \multicolumn{10}{|c|}{ Proposta 1} \\
\hline DMU & $\mathrm{x}_{M C D T}$ & $\mathrm{x}_{M E D}$ & $\mathrm{x}_{R H}$ & $\mathrm{XOC}_{\mathrm{O}}$ & $\mathrm{y}_{\mathrm{CPF}}$ & $\mathrm{y}_{C S M}$ & $\mathrm{y}_{C S I}$ & $\mathrm{y}_{C S A}$ & $\mathrm{y} \operatorname{css}$ & $\mathrm{y} S Q$ \\
\hline 1 & 311190 & 1114935 & 1285500 & 10415 & 845 & 462 & 5133 & 25380 & 17149 & 92 \\
\hline 2 & 350315 & 1192330 & 1050558 & 85950 & 1741 & 610 & 4644 & 29710 & 14783 & 81.8 \\
\hline 3 & 303800 & 1113734 & 1250900 & 50750 & 1780 & 472 & 3230 & 20340 & 15500 & 89.7 \\
\hline 4 & 277140 & 1311200 & 1001297 & 145745 & 1628 & 605 & 4934 & 20422 & 13811 & 83.6 \\
\hline 5 & 232565 & 1147295 & 970000 & 105000 & 732 & 573 & 3013 & 25570 & 19028 & 69 \\
\hline 6 & 362000 & 1622900 & 1285000 & 110900 & 2534 & 782 & 5068 & 29422 & 18854 & 86.6 \\
\hline 7 & 1000840 & 3462876 & 2009990 & 288090 & 3726 & 1081 & 8073 & 54200 & 40307 & 83.4 \\
\hline 8 & 414500 & 1490900 & 1659300 & 335300 & 2641 & 1142 & 4530 & 41247 & 27858 & 90.6 \\
\hline \multicolumn{11}{|l|}{9} \\
\hline 10 & 342900 & 1855000 & 981100 & 97400 & 3353 & 662 & 5068 & 29422 & 18854 & 72.8 \\
\hline 11 & 763900 & 2672000 & 1658890 & 301300 & 4763 & 1084 & 6587 & 42838 & 30037 & 84.0 \\
\hline \multirow[t]{2}{*}{12} & 432500 & 1869000 & 1689900 & 166100 & 3087 & 1052 & 5328 & 35475 & 25593 & 85.2 \\
\hline & \multicolumn{10}{|c|}{ Proposta 2} \\
\hline $\mathrm{DMU}$ & $\mathrm{x}_{M C D T}$ & $\mathrm{x}_{M E D}$ & $\mathrm{x}_{R H}$ & $\mathrm{X}_{\mathrm{OC}}$ & $\mathrm{y}_{\mathrm{CPF}}$ & $\mathrm{y}_{C S M}$ & $\mathrm{y}_{C S I}$ & $\mathrm{y}_{C S A}$ & $\mathrm{y}_{\operatorname{css}}$ & ysQ \\
\hline 1 & 344500 & 1068900 & 1358900 & 115650 & 1048 & 371 & 5308 & 26710 & 18740 & 92.7 \\
\hline 2 & 381560 & 1020700 & 942490 & 101690 & 1207 & 488 & 4190 & 26300 & 14780 & 78.8 \\
\hline 3 & 332600 & 846100 & 1316900 & 61750 & 1420 & 386 & 2680 & 17100 & 12700 & 87.8 \\
\hline 4 & 274440 & 1288720 & 996200 & 150430 & 1666 & 612 & 4964 & 19667 & 14132 & 83.2 \\
\hline 5 & 203699 & 1030610 & 1022900 & 115990 & 1254 & 486 & 2453 & 22522 & 18854 & 66.6 \\
\hline 6 & 472200 & 2010000 & 1487990 & 160900 & 3242 & 960 & 5957 & 36100 & 25080 & 81.3 \\
\hline 7 & 1153760 & 3887255 & 2187990 & 324100 & 4085 & 1153 & 8556 & 56760 & 43700 & 80.5 \\
\hline 8 & 539200 & 1850900 & 1923300 & 402800 & 3351 & 1275 & 5415 & 47890 & 34490 & 85.3 \\
\hline \multicolumn{11}{|l|}{9} \\
\hline 10 & 455000 & 2342000 & 1149100 & 140100 & 4090 & 855 & 6030 & 36610 & 26280 & 65.6 \\
\hline 11 & 878540 & 2996000 & 1790990 & 336700 & 5040 & 1153 & 7052 & 46330 & 33580 & 81.2 \\
\hline \multirow[t]{2}{*}{12} & 553490 & 2287190 & 1939300 & 221100 & 3757 & 1177 & 6160 & 41710 & 31880 & 80.2 \\
\hline & \multicolumn{10}{|c|}{ Proposta 3} \\
\hline DMU & $\mathrm{X}_{M C D T}$ & $\mathrm{X}_{M E D}$ & $\mathrm{X}_{R H}$ & XOC & УCPF & $\mathrm{y} C S M$ & $\mathrm{y} C S I$ & $\mathrm{y}_{C S A}$ & $\mathrm{y} \operatorname{css}$ & ysQ \\
\hline 3 & 380500 & 1000900 & 1000000 & 101690 & 12175 & 489 & 4210 & 21340 & 10789 & 86.3 \\
\hline 6 & 471200 & 2000000 & 1488010 & 160990 & 3249 & 800 & 5500 & 36190 & 25120 & 82.3 \\
\hline 10 & 454890 & 2341090 & 1149090 & 140000 & 4150 & 800 & 5500 & 36623 & 26340 & 70 \\
\hline
\end{tabular}

As propostas de melhoria para o modelo $1 \mathrm{em}$ todos os cenários referidos são apresentadas na Tabela 9, com os fatores que não foram alterados ou estão limitados (proposta 3) mostrados em negrito. 
O GD ficou muito satisfeito com a possibilidade de escolher, para cada caso, a proposta de melhoria que entende ser a mais adequada, uma vez que conhece bem as características específicas de cada unidade.

Para o modelo 2, e atendendo às unidades em avaliação e aos fatores em causa, o GD entendeu suficiente a apresentação apenas das propostas 1 e 2 (Tabela 10).

Tabela 10. Propostas de melhoria para as unidades ineficientes nas escalas originais (modelo 2).

\begin{tabular}{|c|c|c|c|c|c|c|c|c|c|}
\hline \multirow[b]{2}{*}{$\mathrm{DMU}$} & \multicolumn{9}{|c|}{ Proposta 1} \\
\hline & $\mathrm{X}_{M C D T}$ & $\mathrm{X}_{M E D}$ & $\mathrm{x}_{R H}$ & $\mathrm{x}_{O C}$ & $\mathrm{y}_{D M}$ & $\mathrm{y}_{C M}$ & $\mathrm{y}_{D E}$ & $\mathrm{y}_{C E}$ & $\mathrm{y}_{s Q}$ \\
\hline \multicolumn{10}{|l|}{1} \\
\hline 2 & 376000 & 103900 & 1050558 & 99900 & 418 & 57000 & 2100 & 26480 & 79.3 \\
\hline 3 & 298200 & 1113734 & 1240300 & 101750 & 470 & 48580 & 2280 & 24500 & 90 \\
\hline 4 & 277140 & 1232500 & 1001297 & 134300 & 380 & 45000 & 2260 & 19600 & 84.7 \\
\hline 5 & 232565 & 1147295 & 976900 & 106700 & 183 & 40850 & 1480 & 27328 & 68.6 \\
\hline 6 & 339500 & 1535900 & 1238000 & 103300 & 616 & 50768 & 3645 & 34610 & 80 \\
\hline 7 & 636500 & 2737000 & 1569100 & 183100 & 897 & 96530 & 4166 & 41090 & 92.3 \\
\hline 8 & 316100 & 1195900 & 1426900 & 266500 & 712 & 68599 & 3328 & 34052 & 94.8 \\
\hline \multicolumn{10}{|l|}{9} \\
\hline 10 & 319300 & 1753900 & 1348651 & 88700 & 672 & 92720 & 2249 & 45119 & 74.7 \\
\hline 11 & 516690 & 19339800 & 1332000 & 208600 & 758 & 75904 & 5031 & 35040 & 89.6 \\
\hline \multirow[t]{2}{*}{12} & 347800 & 1566900 & 1498400 & 119580 & 707 & 64165 & 3878 & 34831 & 89.6 \\
\hline & \multicolumn{9}{|c|}{ Proposta 2} \\
\hline DMU & $\mathrm{X}_{M C D T}$ & $\mathrm{x}_{M E D}$ & $\mathrm{x}_{R H}$ & $\mathrm{x}_{O C}$ & $\mathrm{y}_{D M}$ & $\mathrm{y}_{C M}$ & $\mathrm{y}_{D E}$ & $\mathrm{y}_{C E}$ & $\mathrm{y}_{s Q}$ \\
\hline \multicolumn{10}{|l|}{1} \\
\hline 2 & 389790 & 1037990 & 953800 & 104500 & 384 & 53480 & 1972 & 25490 & 78.4 \\
\hline 3 & 334900 & 851090 & 1323400 & 62470 & 372 & 38080 & 1910 & 21200 & 87.7 \\
\hline 4 & 262985 & 126600 & 971500 & 141580 & 352 & 43000 & 2150 & 18690 & 84.1 \\
\hline 5 & 206100 & 1042500 & 1031000 & 117900 & 105 & 32290 & 1180 & 30120 & 66.2 \\
\hline 6 & 412800 & 1809000 & 1384000 & 132000 & 515 & 79760 & 4645 & 42560 & 84 \\
\hline 7 & 749500 & 2392000 & 1719100 & 219000 & 841 & 126500 & 5585 & 37750 & 89.2 \\
\hline 8 & 377000 & 13839000 & 1575400 & 311500 & 622 & 107860 & 2874 & 45598 & 92.4 \\
\hline \multicolumn{10}{|l|}{9} \\
\hline 10 & 394800 & 2083000 & 1061700 & 114200 & 562 & 78320 & 3385 & 50740 & 69.2 \\
\hline 11 & 644190 & 2332800 & 1507000 & 258600 & 645 & 104500 & 5938 & 43600 & 87.4 \\
\hline 12 & 441590 & 1899900 & 1709000 & 171190 & 587 & 96100 & 5080 & 43880 & 84.8 \\
\hline
\end{tabular}




\section{Considerações Finais}

Este estudo relata um processo interativo em que o analista e especialistas em saúde trabalharam juntos na avaliação de unidades de CSP, com o intuito de identificar as melhores práticas e contribuir para uma tomada de decisão informada. A abordagem DEA/MCDA proposta anteriormente pelos autores foi utilizada como base de modelação e posteriormente adaptada aos requisitos desta aplicação, ou seja, para definir alvos realistas (com a introdução de um novo parâmetro no modelo) no sentido de possibilitar a ordenação total das DMUs.

A intervenção para avaliar o desempenho das unidades de saúde começa com a escolha pelo GD dos fatores a serem incluídos no modelo, abarcando quatro grandes áreas: acesso, desempenho assistencial, qualidade percecionada e desempenho económico. Fruto das discussões, resultaram dois modelos para avaliar as unidades de CSP. A incorporação das preferências do GD nesses modelos foi possível graças à conversão dos fatores inputs e outputs em funções de utilidade, o que exigiu interpretar estas funções de utilidade como dispositivos para comparar a variação de mérito que corresponde a diferenças de desempenho. Os modelos tornam-se mais completos e credíveis para o GD apenas com a introdução de restrições de coeficientes de ponderação que foram obtidas recorrendo à técnica dos swings para estabelecer uma ordenação dos coeficientes de ponderação. Além disso, foi usada uma questão baseada em trade-offs para limitar a relação entre os coeficientes de ponderação posicionados no primeiro e último lugares e, desta forma, evitar coeficientes de ponderação nulos.

Para cada modelo obteve-se o ranking completo das unidades e foram identificados os pares que lhes serviriam de referência. As propostas de melhoria para as unidades ineficientes surgiram aproveitando o conhecimento do GD sobre as unidades, a fim de obter alvos mais realistas. Para este efeito, um novo modelo foi desenvolvido onde é introduzido um novo parâmetro que limita o valor que uma folga pode ter, possibilitando propostas de melhoria mais ajustadas às preferências do GD.

Este trabalho permitiu facultar informação ao GD no sentido da identificação das melhores práticas, fontes de ineficiência e diferenças em 
relação às melhores práticas, bem como possibilidades e oportunidades de melhoria. Os resultados foram úteis não só para a conceção de medidas corretivas, mas também para informar as decisões sobre os objetivos dos IPs no futuro. Todo o processo de avaliação das unidades de CSP desenrolou-se alternando reuniões de discussão e apresentação de resultados com desenvolvimento metodológicos e testes computacionais, podendo este não ser um processo replicável em todos os casos. Os desenvolvimentos futuros deste trabalho incluem a implementação de um sistema de apoio à decisão que responda em tempo real aos requisitos dos utilizadores.

\section{BIBLIOGRAFIA}

ALI, A.I., \& Seiford, L. (1993) The mathematical programming approach to efficiency analysis. In Fried, Lovell \& Schmidt (Orgs.). The measurement of productive efficiency: techniques and applications (pp.120-113). New York: Oxford University.

ALI, A.I., Lerme, C.S., \& Seiford, L. (1995). Components of efficiency evaluation in Data Envelopment Analysis. European Journal of Operational Research, 80, 462-473.

ALlEN, R., Athanassopoulos, A., Dyson, R.J., \& Thanassoulis, E. (1997). Weights restrictions and value judgments in Data Envelopment Analysis: evolution, development and future directions. Annals of Operations Research, 73,13-34.

ALMEIDA, P.N., \& Dias, L.C. (2012). Value-based DEA models: application-driven developments. Journal of the Operational Research Society, 63, 16-27.

AMADO, C.A.F., \& Dyson, R.G. (2008). On comparing the performance of primary care providers. European Journal of Operational Research, 185, 915-932.

AMADO, C.A.F., \& Santos, S.P. (2009). Challenges for performance assessment and improvement in primary health care: The case of the Portuguese health centres. Health Policy, 91, $43-56$.

ANDERSEN, P., \& Petersen, N.C. (1993). A procedure for ranking efficient units in data envelopment analysis. Management Science, 39, 1261-1264.

ATHANASSOPOUlOS, A.D., \& Podinovski, V.V. (1997). Dominance and potential optimality multiple criteria models decision analysis with imprecise information. Journal of the Operational Research Society, 48 (1), 142-150.

BAKER, R. (1992). General practice in Gloucestershire, Avon and Somerset: Explaining variations in standards. British Journal of General Practice, 42, 415-418.

BANKER, R.D., Charnes, A., \& Cooper, W.W. (1994). Some models estimating technical and scale inefficiencies in Data Envelopment Analysis. Management Science, 3 (9), 1078-1092.

CAMPBELl, S.M., Roland, M.O., Quayle, J.A., Buetow, S.A., \& Shekelle, P.G. (1998). Quality indicators for general practice: Which ones can general practitioners and health authority managers agree are important and how useful are they?. Journal of Public Health Medicine, 20, 414-421.

CHARNES, A., Cooper, W.W., \& Rhodes, E. (1978). Measuring the efficiency of decision making units. European Journal of Operational Research, 2 (6), 429-444. 
CharneS, A., Cooper W.W., Golany, B., Seiford, L., \& Stutz, J. (1995). Foundations of Data Envelopment Analysis for Pareto-Koopmans efficient empirical production functions. Journal of Econometrics, 30, 91-107.

Avaliação sobre o funcionamento das Unidades de Saúde do ACES - BM1. (2010). Coimbra.

Coodenação Estratégica dos CSP. (2010). Metodologia de Contratualização, Administração Central do Sistema de Saúde.

DIAS, L.C., \& Clímaco, J.N. (2000). Additive Aggregation with Variable Interdependent Parameters: the VIP Analysis Software. Journal of the Operational Research Society, 51 (9), 107010822.

DONABEDIAN, A. (1980). The Definition of Quality and Approaches to its Assessment. Ann Arbor, MI, Health Administration Press.

DULÁ, J.H., \& Hickman, B.L. (1997). Effects for excluding the column being scored from DEA envelopment LP technology matrix. Journal of the Operational Research Society, 48, 1001-1012.

DYSOn, R.G., Allen, R., Camanho, A.S., Podinovski, V.V., Sarrico, C.S. \& Shale, E.A. (2001). Pitfalls and protocols in DEA. European Journal of Operational Research, 132, 245-259.

FARRELL, M.J. (1997). The Measurement of productive efficiency. Journal of Royal Statistical Society, 120 (A), 353-290.

GIUFFrIDA, A., Gravelle, H., \& Sutton, M. (2000). Efficiency and administrative costs in primary care. Journal of Health Economics, 19 (6), 983-1006.

GOODWIN, P., \& Wright, G. (1998). Decision analysis for management judgment. Wiley: Chichester, 1998.

GOUVEIA, M.C., Dias, L.C., \& Antunes, C.H. (2008). Additive DEA based on MCDA with imprecise information. Journal of the Operational Research Society, 59, 54-63.

GOUVEIA, M.C., Dias, L.C., \& Antunes, C.H. (2013). Super-efficiency and stability intervals in additive DEA. Journal of the Operational Research Society, 64, 86-96.

HOLlingswORTH, B. (2003). Non-Parametric and Parametric Applications Measuring Efficiency in Health Care. Health Care Management Science, 6, 203-218.

HUANG, Y.G., \& McLaughlin, C.P. (1989). Relative efficiency in rural primary health care: an application of data envelopment analysis. Health Services Research, 24, 143-158.

Instituto de Gestão Informática e Financeira da Saúde. (2006). Estatística do Movimento Assistencial dos Centros de Saúde em 2005.

KEENEY, R.L., Raiffa, H. (1976). Decisions with multiple objectives: preferences and value tradeoff. Wiley: New York.

KIRIGIA, J.M., Emrouznejad, A., Sambo, L.G., Munguti, N., \& Liambila, W. (2004). Using data envelopment analysis to measure the technical efficiency of public health centers in Kenya. Journal of Medical Systems, 28, 155-166.

LINNA, M., Nordblad, A., \& Koivu, M. (2003). Technical and cost efficiency of oral health care provision in Finnish health centres. Social Science \& Medicine, 56, 343-353.

Milliken, O., Devlin, R.A., Barham, V., Hogg, W., Dahrouge, S., \& Russell, G. (2011). Comparative Efficiency Assessment of Primary Care Service Delivery Models Using Data Envelopment Analysis. Canadian Public Policy, 37, 85-109.

MCCOLL, A., Roderick, P., Gabbay, J., Smith, H., Moore, M. (1998). Performance indicators for primary care groups: An evidence based approach. British Medical Journal, 317, 1354-1360. 
NUTI, S., Daraio, C., Speroni, C., \& Vainieri, M. (2011). Relationships between technical efficiency and the quality and costs of health care in Italy. International Journal for Quality in Health Care, 23, 324-330.

Organização para a Cooperação e Desenvolvimento Económico. (2006). Projecting OECD bealth and long term care expenditures: what are the main drivers? Economics Department. Working Papers 477.

Observatóro Português dos Sistemas de Saúde. (2007). Relatório de Primavera 2007. Luzes e sombras: a governação da saúde, Coimbra: Mar da Palavra.

Observatóro Português dos Sistemas de Saúde. (2008). Relatório de Primavera 2008. Sistema de Saúde Português - Riscos e Incertezas, Coimbra: CEISUC.

OZCAN, Y.A. (1993). Sensitivity Analysis of Hospital Efficiency under Alternative Output/Input Combinations and Peer Groupings. International Journal of Knowledge and Policy, 4, 1-31.

PROCTOR, S., \& Campbell, J. (1999). A developmental performance framework for primary care. International Journal of Health Quality Assurance, 12 (7), 279-286.

PODINOVSKI, V.V. (2004). Production trade-offs and weight restrictions in data envelopment analysis. Journal of the Operational Research Society, 55 (12), 1311-22.

ROSENMAN, R., \& Friesner, D. (2004). Scope and scale inefficiencies in physician practices. Health Economics, 13, 1091-1116.

ROUSE, P., Harrison, J., \& Turner, N. (2011). Cost and Performance: Complements for Improvement. Journal of Medical Systems, 35, 1063-1074.

SALINAS-JIMENEZ, J., \& Smith, P. (1996). Data envelopment analysis applied to quality in primary health care. Annals of Operational Research, 67, 141-161.

SALO, A.A., \& Hämäläinen, R.P. (2001). Preference Ratios in Multiattribute Evaluation (PRIME) elicitation and decision procedures under incomplete information, IEEE Transactions on Systems Man and Cybernetics part A, 31, 533-545.

THANASSOUlIS, E., Boussofiane, A., \& Dyson, R.G. (1996). A comparison of data envelopment analysis and ratio analysis as tools for performance assessment. Omega, 24, 229-244.

THANASSOUlIS, E., Portela, M.C., Allen, R. (2004). Incorporating value judgments in DEA. In Cooper, W.W., Seiford, L.M., Zhu, J. (eds.), Handbook on Data Envelopment Analysis (pp.99-137). Boston: Kluwer.

THANASSOUlis, E., Portela, M.C., Graveney, M. (2012). Estimating the scope for savings in referrals and drug prescription costs in the General Practice units of a UK Primary Care Trust. European Journal of Operational Research, 221, 432-444.

VON WINTERFELDT, D., Edwards, W. (1986). Decision analysis behavioural research. New York: Cambridge University Press.

VAN DEN HOMBERGH, P., Grol, R., van den Hoogen, H.J.M., \& van den Bosch, W.J.H.M. (1998). Assessment of management in general practice: Validation of a practice visit method. British Journal of General Practice, 48, 1743-1750.

WAGNER, J., Shimshak, D., Novak, M. (2003). Advances in physician profiling: the use of DEA. Socio-Economic Planning Sciences, 37, 141-163. 